\title{
Fracturas de la Unidad Radiocubital Distal
}

\author{
G. Celester Barreiro ${ }^{(\mathrm{I})}$, B. Masip BilbaO ${ }^{(2)}$, \\ A. Prego Bestilleiro ${ }^{(3)}$, J. M. Dozo García ${ }^{(3)}$. \\ (1) Unidad de Cirugía de la Mano del Hospital Modelo. Mutua la Fraternidad. a Coruña. \\ (2) Servicio de C.O.T. Complexo Hospitalario de Pontevedra \\ (3) Mutua Gallega. Hospital Santa Teresa. A Coruña.
}

Correspondencia:

gcelester@fraternidad.com

\section{INTRODUCCIÓN}

S e ha tomado el título de fracturas de la unidad radiocubital distal, como le gustaba llamarlas al profesor Gomar ${ }^{1}$, y no el habitual de fracturas de la extremidad del radio, por entender que refleja mejor el compendio de las lesiones sufridas en las fracturas distales del antebrazo, si bien la mayor parte del presente artículo pretende revisar la patología fracturaria de esta zona del radio.

Las fracturas de la extremidad distal del radio (FEDR) son las más frecuentes del esqueleto después de las fracturas de las falanges de la mano en su conjunto y hasta hace muy pocos años se consideraba que su resultado funcional era habitualmente bueno aun en ausencia de tratamiento o cuando éste no había conseguido una aceptable reducción anatómica. Muchos malos resultados, amén de mucha yatrogenia y desidia médica, se apoyaron en la desafortunada observación del propio Abraham Colles ${ }^{2}$ de que "si la fractura queda sin tratar desaparecerá el dolor en un determinado período de tiempo y la movilidad de recuperará, si bien la deformidad permanecerá toda la vida".

Ciertamente, gran parte de los pacientes que han sufrido una FEDR recuperan el uso de la mano y pueden realizar la mayoría de sus actividades domésticas y laborales sin demasiada dificultad. Pero no es así en pacientes jóvenes que han sufrido un traumatismo de alta energía $\mathrm{y}$ tiene altas exigencias funcionales ${ }^{3}$. La mejora de los medios de exploración, de tratamiento y los criterios de calidad actuales nos obligan a ser cada vez más rigurosos y tomar por malos resultados muchos de los que hace apenas dos décadas daríamos por buenos.

En los últimos años, con los mejores conocimientos de la anatomía patológica de las FEDR, los modernos medios de imagen (fluoroscopia, TAC, RM, artroscopia) y la aparición del nuevo material de osteosíntesis (múltiples fijadores externos, placas de formas diversas y tornillos canulados de doble rosca), junto con la mayor demanda social de buenos resultados, han cambiado radicalmente la actitud del cirujano con respecto a estas lesiones, de tal modo que en la última década parece haberse impuesto el tratamiento quirúrgico para gran parte de estas fracturas. No obstante, debido a las tendencias de moda por la presión de las casas comerciales y la rentabilización de tiempo en consultas, ya que el tratamiento conservador conlleva un exigente seguimiento clínico, tal vez estemos abusando de los métodos a cielo abierto en muchas fracturas que bien podrían tratarse de forma ortopédica o con fijación percutánea con dos simples y baratas agujas de Kirschner.

\section{PERSPECTIVA HISTÓRICA}

\section{II.1. Diagnóstico:}

Dada la alta incidencia de las FEDR, es lógico pensar que fueron conocidas y tratadas por los médicos o sanadores de todas las épocas y cultu- 
ras, pero no deja de ser curioso el hecho de que hasta finales del siglo XVIII se creyese que no se trataba de una fractura sino de una luxación de la muñeca, sin duda debido a que estas fracturas presentan algunas peculiaridades con respecto a otras, como la ausencia de crepitación y de movilidad en el foco, que hoy sabemos debido a la impactación de los fragmentos ${ }^{4}$.

Este error, debido en gran parte al seguimiento de la autoridad de Hipócrates, que en el siglo IV antes de Cristo las definió como una dislocación de la muñeca, fue transmitido a lo largo de dos mil años a través de los escritos de Galeno, Paracelso y otros médicos medievales y renacentistas.

En nuestro entorno cultural, probablemente fue JL Petit ${ }^{5}$, a finales del siglo XVIII, el primero en sugerir que se traba de una fractura y no de una luxación. En el mismo siglo, Pouteau ${ }^{6}$ llegó a la misma conclusión y describió el desplazamiento habitual de los fragmentos en un trabajo que se publicó después de su muerte en 1783. Durante casi treinta años no consta en la literatura médica ningún avance ni en la descripción ni en el tratamiento de estas fracturas, hasta que en 1814 aparece el famoso artículo de Abraham Colles $^{2}$ en el que describe la lesión como "una fractura de la extremidad distal del radio que ocurre a una pulgada y media de la articulación carpiana", así como el desplazamiento y algunas pautas de tratamiento.

Sin embargo, Abraham Colles nunca pudo comprobar en cadáveres la validez de sus afirmaciones. Sería Dupuytren ${ }^{7}$ quien pocos años más tarde comprobaría en estudios postmortem la morfología de estas fracturas, y casi simultáneamente con la publicación de estas observaciones aparece un trabajo de su coterráneo Malgaigne ${ }^{8}$ identificando gran variedad de deformidades asociadas y la frecuencia de estas fracturas en los hospitalizados en el Hôtel Dieu, en el que podemos considerar como el primer estudio estadístico de la historia sobre las FEDR. Malgaigne además fue el primero en sospechar que a distintas posiciones de la mano en la caída correspondían distintos desplazamientos de la fractura.

Otro cirujano francés, Goyrand ${ }^{9}$, en 1832, confirmó el característico desplazamiento dorsal del fragmento distal del radio, pero observó que en algunos casos el desplazamiento tiene lugar en dirección volar. RW Smith ${ }^{10}$, de Dublín, describió en 1847 las fracturas con este mismo desplazamiento achacándolo a la caída sobre el dorso de la mano. Desde entonces, este tipo de lesión se conoce con el epónimo de ambos: fractura de Goyrand-Smith.

En 1838 Rhea Barton ${ }^{11}$ publicó la descripción de la fractura marginal posterior, que hoy lleva su nombre, y la anterior, o fractura de Rhea Barton inversa.

\subsection{Tratamiento:}

Fue Abraham Colles el primero en describir la maniobra de reducción con las dos manos del cirujano y la ulterior inmovilización con dos férulas de madera desde el codo hasta la raíz de los dedos. No tenemos constancia de cómo se trataban estas fracturas antes del siglo XIX; es de suponer que con un vendaje de compresas y entablillado simple, como las demás fracturas de los miembros. Pero en este siglo numerosos autores interesados en las FEDR diseñaron su propia férula de inmovilización, casi siempre de madera y en algún caso de metal ${ }^{4-11}$.

Pirogov ${ }^{12}$, un cirujano ruso destacado a la guerra de Crimea, fue quien empleó por primera vez el yeso en 1854 para inmovilizar las fracturas en la evacuación de los heridos del frente a la retaguardia. Desde entonces, este fue el material más empleado hasta la actualidad.

\section{ANATOMÍA PATOLÓGICA}

La articulación de la muñeca, parte móvil entre el antebrazo y la mano, está constituida por las porciones epifisarias distales del radio y del cúbito, los huesos del carpo y los medios de unión (cápsula y ligamentos) entre estas estructuras óseas.

Desde un punto de vista funcional puede admitirse la existencia de tres grandes articulaciones (radiocarpiana, mediocarpiana y radiocubital distal) y las FEDR afectan al funcionamiento de todas ellas. Las dos primeras intervienen en los movimientos de flexión, extensión y desviaciones laterales; la última en los movimientos de pronosupinación.

La extremidad metafisoepifisaria distal del radio está formada por hueso esponjoso y presenta, 
en una sección transversal, la forma de una pirámide con cuatro caras, una base y un ancho borde. La cara proximal es la continuación de la diáfisis del hueso. La cara dorsal, convexa, está en íntimo contacto con los tendones extensores que la cruzan; la cara volar, ligeramente cóncava, da asiento al músculo pronator quadratus; la cara interna contiene en toda su extensión una carilla articular, la cavidad sigmoidea, para articularse con el cúbito. La base, o glena radial, recubierta de cartílago, tiene en la unión de su tercio interno con los dos tercios externos una suave cresta anteroposterior que la divide en dos facetas, una interna, cuadrada, para el semilunar, y otra externa, triangular, para el escafoides. El robusto borde, constituido por la apófisis estiloides del radio, presta inserción al brachioradialis y tiene un surco para los tendones del primer compartimento dorsal.

La glena radial presenta, con respecto a la diáfisis del hueso, una inclinación radio-cubital de $21^{\circ}$ a $23^{\circ}$ y una inclinación dorso-volar de $10^{\circ}$ a $12^{\circ}$. Es fundamental tener presente esta disposición para valorar los desplazamientos fracturarios y su correcta reducción.

El 60\% de las FEDR son articulares (en V o $\mathrm{Y}$, marginales anterior, posterior, externa, interna o conminutas). La posibilidad de desplazamiento de los fragmentos es diversa y su reducción exige con frecuencia tratamiento cruento. Las fracturas extraarticulares $(40 \%)$ pueden cursar con un desplazamiento dorsal del fragmento distal (fracturas de Colles) ${ }^{2}$ o, más rara vez, con desplazamiento volar (fractura de Smith ${ }^{10}$.

En la fractura de Colles el fragmento distal suele desplazarse en los tres planos del espacio: hacia atrás (dorsal), lateral (radial), proximal (impactación) y en rotación (supinación).

\section{DIAGNÓSTICO}

\section{IV.1. Diagnóstico clínico:}

Aunque el diagnóstico de las FEDR debe ser fundamentalmente radiológico, ante un traumatismo de la muñeca no debe obviarse nunca la anamnesis y la exploración clínica. Se valorará el mecanismo (golpe directo, caída, torsión, aplastamiento), tiempo de evolución, edad, actividad del paciente, etc.
En la exploración clínica, además del edema e impotencia funcional habituales en toda fractura, podrá apreciarse que la de Colles típica presenta unas deformidades que se corresponden con el desplazamiento fracturario descrito en el apartado anterior. El desplazamiento dorsal y la impactación del fragmento distal harán que el radio se acorte y que su apófisis estiloides quede al mismo nivel que la apófisis estiloides del cúbito (signo de Laugier). Cuando la muñeca se ve de lado tiene el característico aspecto de "dorso de tenedor" con una prominencia dorsal debido al fragmento distal desplazado, y una prominencia volar, más redondeada, debido al extremo proximal empujando los tendones flexores hacia delante. Las luxaciones del carpo presentan una deformidad similar pero más distal y con la movilidad de los dedos bloqueada, lo que no ocurre en la fractura de Colles.

El desplazamiento radial del fragmento distal hace que toda la mano acompañe radialmente a este fragmento dando el típico aspecto "en bayoneta".

\section{IV.2. Diagnóstico radiológico:}

Es fundamental tener una buena concepción espacial del grado de desplazamiento y de la conminución de los fragmentos antes de indicar el tipo de tratamiento. La radiología simple es la prueba diagnóstica por antonomasia en este tipo de lesiones y casi siempre nos permitirá valorar correctamente ambos parámetros y la densidad ósea. No obstante, en algunas fracturas articulares y conminutas el estudio debe completarse con una TAC, tomografías computarizadas tridimensionales y en algún caso particular con la visión artroscópica, que además nos ayudará en la reducción de pequeños fragmentos.

Con respecto a las radiografías se debe insistir en la calidad de contraste para ver todos los posibles fragmentos y que las proyecciones sean perfectas tanto en la posición antero-posterior, que nos permitirá valorar la inclinación lateral del fragmento distal del radio, si hay acortamiento y la posible afectación de la estiloides cubital, como en la lateral para observar la inclinación volar. En caso de duda son de gran ayuda las proyecciones oblicuas.

En las fracturas extraarticulares debe valorarse el acortamiento y la orientación de la glena en sus inclinaciones radio-cubital y postero-an- 
terior. En las intraarticulares y en las conminutas hay que fijarse en la dirección del trazo o trazos de fractura, en el número, tamaño de los fragmentos y en su desplazamiento para valorar si es posible su reducción incruenta o si precisarán fijación interna o externa.

Debe observarse además si hay fractura de la apófisis estiloides del cúbito, que de existir puede ser un signo de inestabilidad, y en el estado de la articulación radiocubital distal.

\section{EPIDEMIOLOGÍA}

Las FEDR son muy frecuentes, llegando a representar un $15 \%$ de todas las fracturas tratadas en urgencias ${ }^{13}$. Predominan en tres épocas de la vida: en niños y jóvenes entre 6 y 18 años $(14,4 \%)^{14}$, en los que la línea de fractura pasa a través del cartílago de crecimiento; en el adulto joven, cuya incidencia ha aumentado como consecuencia de accidentes deportivos y de tráfico $(35,6 \%)^{14} ; \mathrm{y}$, las más frecuentes, en pacientes mayores de 60 años $(50 \%)^{14}$, en los que la osteoporosis si bien no es la causa sí supone un factor agravante en un $75 \%$ de los casos.

En cuanto a la incidencia por sexo, en mujeres mayores de 35 años es cuatro veces superior a la de los hombres y su prevalencia aumenta progresivamente en la menopausia en relación con los cambios hormonales y la osteoporosis ${ }^{15}$. Falch et al. ${ }^{16}$ encuentran que entre los 60 y 70 años es cinco veces más frecuente en mujeres, igualándose el porcentaje a los 91 años.

Un estudio de Ahlborg et al. ${ }^{17}$, con seguimiento de 15 años en mujeres postmenopáusicas, ha demostrado que el diámetro intramedular aumenta y el índice de resistencia disminuye en el radio distal proporcionalmente al descenso de los niveles de estradiol. Este mismo estudio señala que el descenso de una desviación estándar en el índice de resistencia produce un aumento de 3,8 en el riesgo de fractura de radio distal, lo que justificaría estas variaciones de edad y sexo.

\section{MECANISMOS DE FRACTURA}

El mecanismo directo, por un golpe sobre la muñeca, es sumamente raro. Lo habitual es que las FEDR desplazadas dorsalmente se deban a un mecanismo indirecto por una caída sobre la mano en hiperextensión transmitiendo la carga a la extremidad distal del radio, lo que produce fuerzas de tensión en la cara volar, fuerzas de compresión en la cara dorsal y supinación del fragmento distal de la fractura.

Según Fernández ${ }^{4,18}$ se pueden distinguir cinco patrones de fractura dependiendo del mecanismo de lesión:

Mecanismo de bisagra: Produce fracturas extraarticulares metafisarias. Una cortical metafisaria falla debido a la fuerza de tensión y la cortical opuesta tiene un cierto grado de conminución. Incluye las fracturas de Colles y Smith.

Mecanismo de cizallamiento: Produce fracturas-luxaciones con trazo intraarticular. Incluye las fracturas de Barton, Barton inversa y de la estiloides radial (fractura del chófer).

Mecanismo de compresión: Se producen fracturas intraarticulares complejas debido al colapso subcondral y metafisario.

Mecanismo de avulsión: Son el resultado de la avulsión de los ligamentos radiocarpianos. Con cierta frecuencia los fragmentos son muy pequeños y se producen fracturas-luxaciones de muñeca inestables y difíciles de sintetizar.

Mecanismo combinado: Suelen deberse a traumatismos de alta energía.

Por tanto, la posición de la mano y la muñeca en el momento de la lesión determinará la zona sometida a una máxima compresión y la modificación de las fuerzas ligamentosas, marcando así el tipo de desplazamiento, el patrón de fractura y que ésta sea extra o intraarticular ${ }^{19}$.

\section{CLASIFICACIÓN}

Existe una amplia terminología para definir los diferentes tipos de FEDR: articulares-extraarticulares, totales-parciales, desplazadas-no desplazadas, estables-inestables, conminutas-no conminutas, reducibles-no reducibles.

Muchos conceptos no están exentos de cierta subjetividad por parte del observador, por lo que desde Abraham Colles han sido numerosísimos los intentos de establecer una clasificación que englobe todos los tipos posibles de fractura y con ello la mejor opción terapéutica para cada modalidad. 
De las diversas clasificaciones de las FEDR algunas tuvieron una vigencia temporal y geográfica, especialmente en sus países de origen; otras continúan empleándose en la actualidad, a veces más por el prestigio de su autor que por haber aportado verdaderas innovaciones anatomopatológicas. Todas se basan en el aspecto radiológico si bien algunas, como las de Castaing et al. ${ }^{20}$ y la de Fernández y Jupiter ${ }^{4}$, se fundamentan en el mecanismo lesional.

Aun cuando no es perfecta, porque no contempla de forma aislada las fracturas marginales internas ni el frecuente fragmento posterointerno, los autores de este trabajo emplean sistemáticamente la clasificación de la AO, propuesta por Fernández y Müller en $1987^{21-22}$, por entender que es sencilla, fácil de recordar y permite registrar casi todas las posibilidades.

\section{TRATAMIENTO}

El objetivo del tratamiento de las FEDR es lograr una muñeca indolora, estable y con la mayor movilidad posible. A menudo es muy difícil conseguir la correcta reducción de las superficies articulares de la muñeca y los tratamientos inadecuados suelen acarrear catastróficos resultados funcionales.

No existe, en la actualidad, un único método óptimo para todas las formas de lesión. Junto con el tipo de fractura debemos valorar la edad y actividad del paciente; estos tres parámetros son los que condicionarán el tratamiento más adecuado para cada caso en particular. Si bien en las fracturas extraarticulares y estables sigue vigente el clásico tratamiento conservador, la búsqueda de la restitutio ad integrum, especialmente en los pacientes jóvenes, hace cada vez más frecuente el tratamiento quirúrgico.

\section{VIII.1 Tratamiento ortopédico:}

El tratamiento conservador de las FEDR no ha cambiado significativamente en los últimos años. Se emplea en las fracturas cerradas por baja energía que cumplen los criterios de estabilidad (acortamiento radial menor de $2 \mathrm{~mm}$, escalón articular menor de $2 \mathrm{~mm}$, angulación volar-dorsal entre $-20^{\circ} \mathrm{y}+10^{\circ}$, angulación radiocubital mayor de $15^{\circ}$ y sin conminución dorsal), en pacientes con baja demanda funcional o cuando resultan estables tras su manipulación.

La reducción, cuando es precisa, es preferible realizarla antes de 8 horas para evitar la tumefacción y bajo anestesia regional con tracción suave pero mantenida para desimpactar los fragmentos. Esta tracción puede ejercerse en horizontal, con dos ayudantes, sin tirones bruscos, o mediante el método de Böhler ${ }^{37}$ de tracción zenital mantenida con una contratracción de $4 \mathrm{Kg}$. durante unos 5 minutos, al cabo de los cuales aplicamos ambos pulgares sobre el fragmento distal llevándolo en flexión, desviación cubital y pronación en las fracturas de Colles. Luego, aun manteniendo la tracción, se comprueba la reducción con el intensificador de imágenes y si cumple los criterios de estabilidad se inmoviliza con escayola antebraquiopalmar moldeada en posición neutra o con ligeros grados de flexión, desviación cubital y pronación. Las posturas de flexión forzada son fuente de numerosas complicaciones. Debe hacerse siempre un control radiológico a los pocos días para confirmar la reducción y revisar periódicamente el yeso, que debe quedar en posición neutra en la tercera semana.

En determinados casos, como en la fractura de Smith, donde existe una deformidad rotatoria, puede ser necesaria la inmovilización en extensión y supinación de $45^{\circ}$ a $60^{\circ}$, con yeso bivalvado braquio-palmar durante 4 semanas, seguido de otro antebraquio-palmar 2 semanas más.

\section{VIII.2 Enclavado percutáneo y tracción bipolar con agujas de Kirschner:}

En muchas fracturas inestables o conminutas el vendaje de escayola puede ser insuficiente para mantener la reducción, aun cuando ésta hubiera sido aceptable tras la tracción y manipulación de los fragmentos. Son varias las causas que pueden ocasionar un redesplazamiento de la fractura; la pérdida de masa ósea en el foco debido a la impactación, el aflojamiento del vendaje al disminuir el edema y la existencia de fragmentos libres, sin unión ligamentosa, son las más frecuentes. En estos casos, para evitar las posiciones de inmovilización forzadas, que son fuente de compresiones nerviosas, síndrome 
doloroso regional complejo y rigidez, es útil la fijación percutánea de los fragmentos con agujas de Kirschner. En ocasiones esta técnica hay que realizarla tras el desplazamiento secundario a los pocos días de haber hecho la reducción, por lo que es conveniente verificar radiológicamente la estabilidad entre el $5^{\circ}$ y el $7^{\circ}$ día. En cualquier caso, se debe prever esta complicación y ofrece más garantías fijar ya de entrada aquellas fracturas que presenten una angulación dorsal mayor de $20^{\circ}$, conminución dorsal, acortamiento radial de $10 \mathrm{~mm}$, escalón articular mayor de $2 \mathrm{~mm}$, fracturas asociadas a luxaciones, con un fragmento posterointerno del margen radial (die punch) y en aquéllas asociadas a fracturas del cúbito $^{23-24}$.

La técnica a emplear dependerá del tipo de fractura y de las preferencias del cirujano, pero siempre mejor con anestesia regional que local, bajo control radiológico y guardando la mayor asepsia posible. Su indicación principal es en fracturas extraarticulares desplazadas, sin conminución y en las articulares simples (C1) en pacientes jóvenes con buena calidad ósea ${ }^{23,25}$.

La biomecánica y eficacia de la fijación percutánea se explica muy bien con la teoría de las tres columnas de carga y estabilidad en la zona metafiso-epifisaria del cúbito y el radio (Figura 1) propuesta por Rikli y Regazzoni en $1996^{26}$. Las fuerzas de compresión axial ejercidas en la

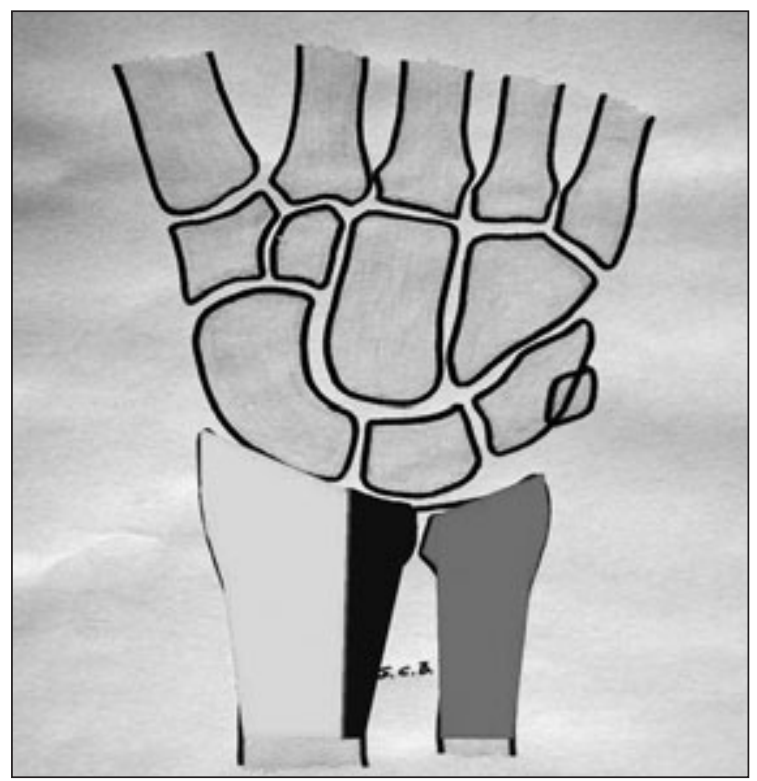

Figura 1: Teoría de las tres columnas de Rikli y Regazzoni $^{26}$. mano se transmitirán a los dos huesos del antebrazo, pero no por igual a cada uno de ellos dependiendo de la posición de la mano. La función de la columna interna, formada por el cúbito y el ligamento triangular, es de estabilidad y transmisión de cargas; la columna intermedia, que contiene las carillas para el semilunar y la cavidad sigmoidea, tiene la misión de soportar cargas, y la columna externa, formada por la estiloides del radio y la carilla para el escafoides, tiene sobre todo funciones de estabilidad.

La mayoría de las técnicas de fijación percutánea buscan fijar al menos dos de las tres columnas.

\section{VIII.2.1. Fijación de las columnas externa y me- dia:}

Desde inicios del siglo XX vienen empleándose las agujas de Kirschner para mantener la reducción introduciendo habitualmente una o dos por la apófisis estiloides del radio; es decir, fijando la columna externa y la cortical radial interna, método que, con numerosas variantes, fue el más empleado hasta la actualidad y resulta excelente para las fracturas de Colles típicas, las de apófisis estiloides radial e incluso para algunas intraarticulares (tipos B1 y $\mathrm{C} 1$ de la $\mathrm{AO}$ ) (Figura 2).

Posteriormente vendrían múltiples propuestas de triangulación con agujas desde la zona distal del borde diafisario externo y la apófisis estiloides hacia la parte interna de la epífisis radial, método que aporta ventajas en las fracturas articulares simples con trazo en $\mathrm{V}_{\text {o }} \mathrm{Y}^{27}$ (Figura 3).

\section{VIII.2.2. Fijación de las columnas interna y ex- terna:}

En 1952, De Palma ${ }^{28}$ propuso la fijación transulnar-estiloradial utilizando la zona diafiso-metafisaria distal del cúbito, íntegra, a modo de fijador interno de la fractura radial. Con una variante de esta técnica, Toledo et al. ${ }^{23}$ publicaron una casuística de 90 pacientes con resultados buenos o excelentes en el $94 \%$. Como esta fijación bloquea la radio-cubital distal, estos autores aconsejan un yeso braquio-palmar durante 3 semanas seguido de uno antebraquio-palmar otras 3.

Debido a la frecuencia de un fragmento postero-interno desplazado en el margen radial $(25 \%)$, que afecta a las dos superficies articula- 

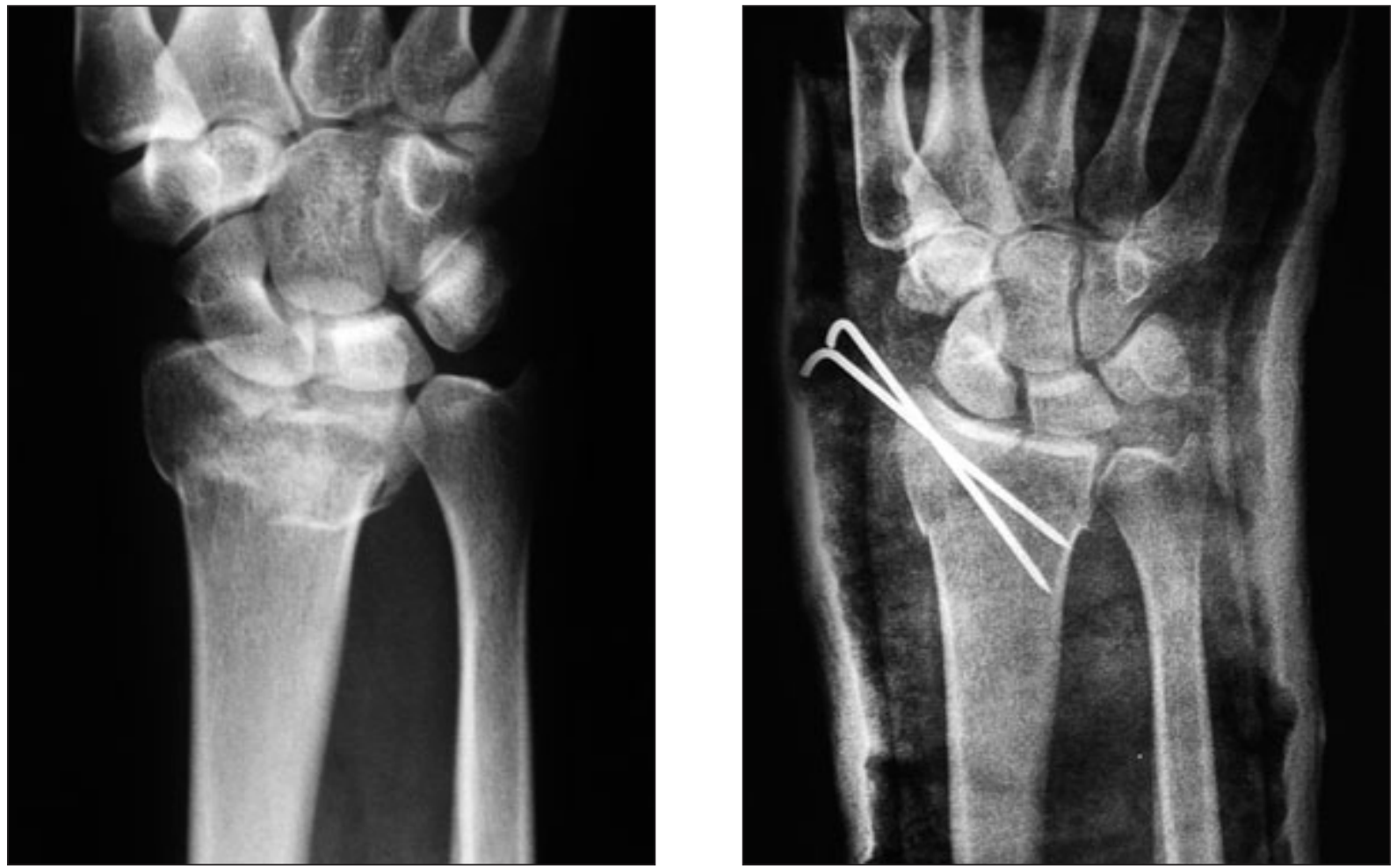

Figura 2: A: Fractura tipo C1. B: Método de fijación más habitual.
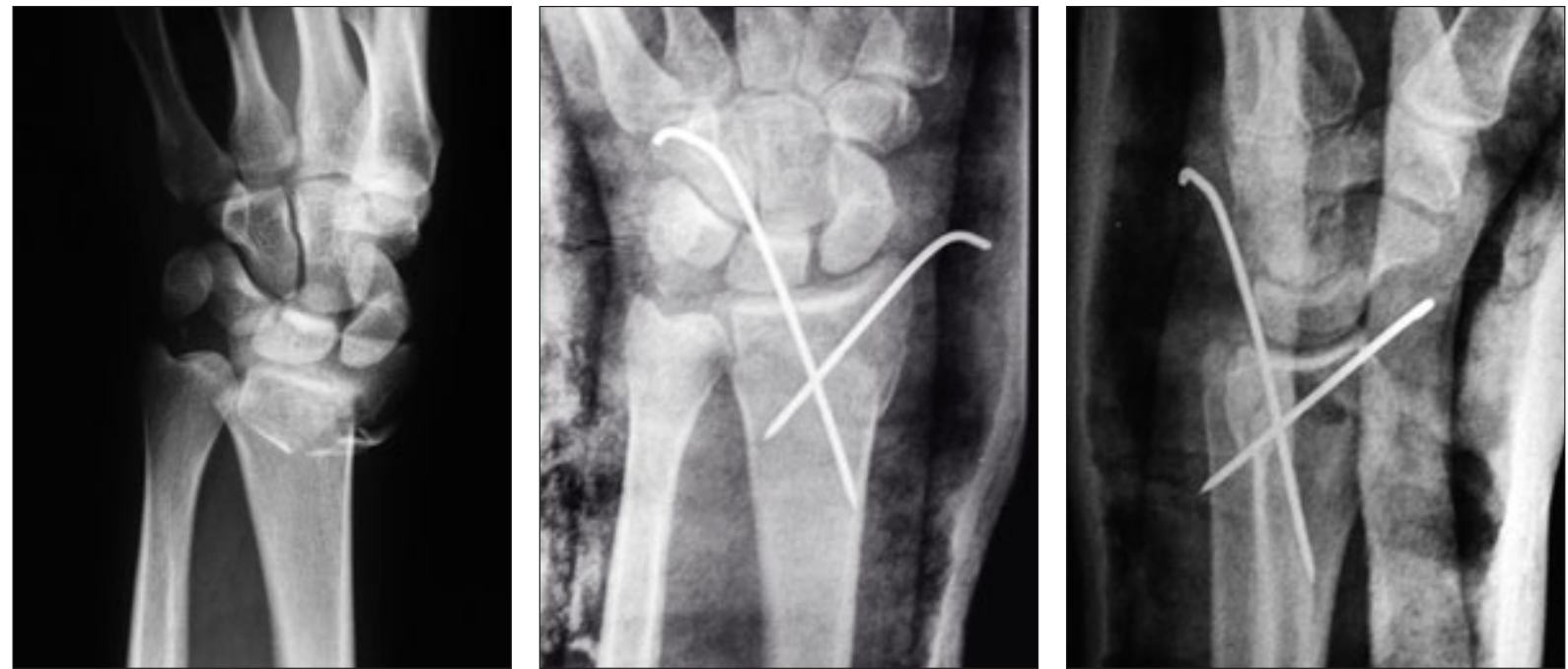

Figura 3: A: Fractura tipo C2. B y C: Fijación con el método de Stein y Katz ${ }^{27}$.

res del radio y suele ser reducible pero inestable, Mortier et al. ${ }^{29}$ propusieron en 1986 el doble enclavado vertical y transversal con dos agujas por la estiloides radial y una transversal fijando las epífisis cubital y radial (Figura 4); y en 1989 Rayhack ${ }^{30}$ publicó su método de enclavado múltiple cúbito-radial con varias agujas (hasta 6) fijando la metáfisis y epífisis de ambos huesos.
VIII.2.3. Fijación intrafocal:

Para las fracturas extraarticulares con desplazamiento dorsal un excelente método de osteosíntesis con agujas resultó el de fijación intrafocal, publicado por Kapandji en $1976^{31}$ pero descrito por el mismo autor tres años antes en las Jornadas Franco-Italianas de Bellagio ${ }^{32}$. Consiste en introducir las agujas directamente en el 

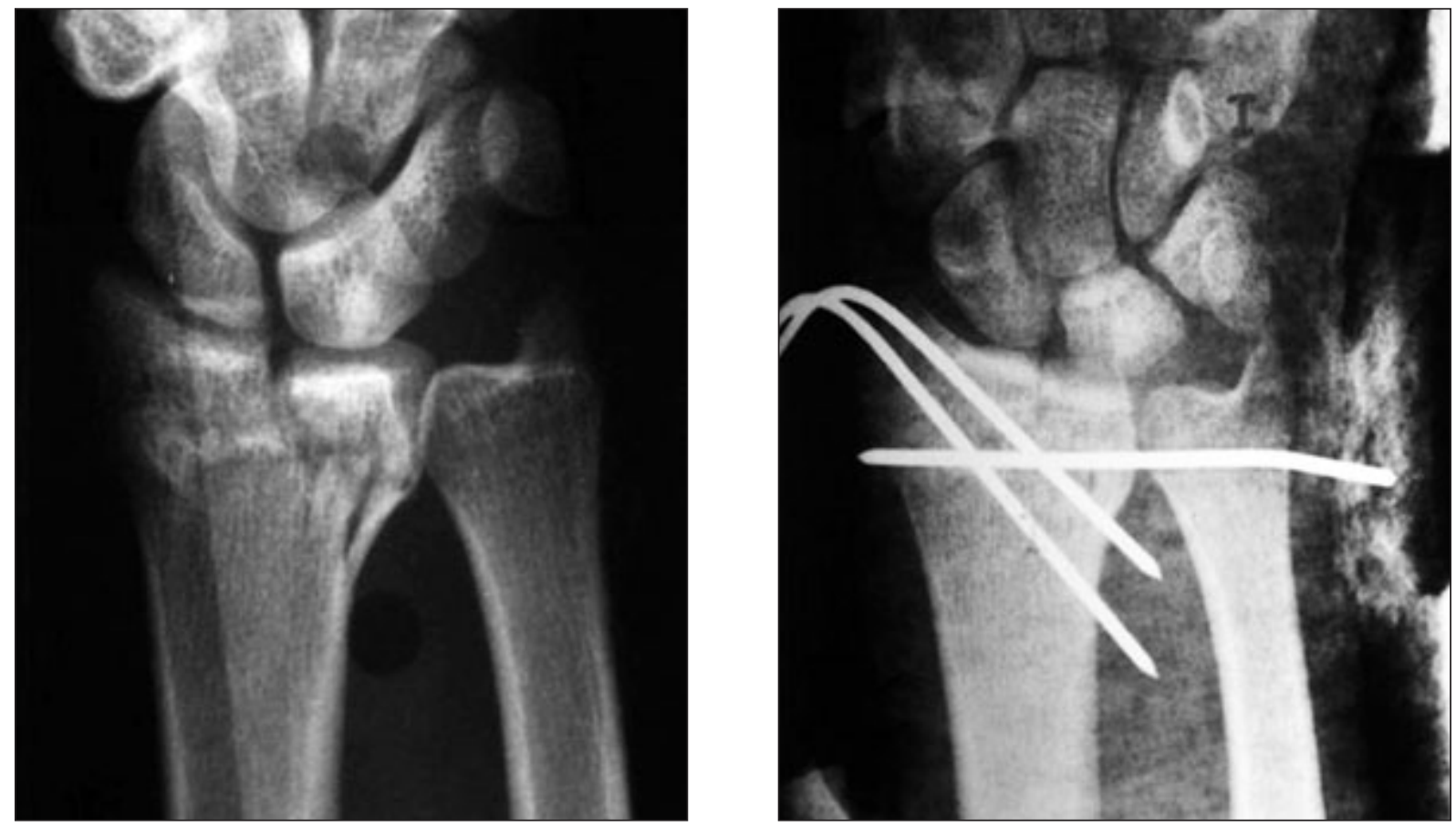

Figura 4: A: Fractura tipo C2. B: Fijación con el método de Mortier et al. ${ }^{29}$

foco de fractura a través de pequeñas incisiones longitudinales de 10 ó $15 \mathrm{~mm}$ sobre la estiloides y cara dorsal del radio. Una vez hecha la reducción, la aguja introducida en el foco se inclina unos $45^{\circ}$ en dirección proximal y se hace progresar hasta fijarla en la cortical opuesta. El talón de las agujas -habitualmente dos o tres- evita el redesplazamiento secundario y puede permitirse una movilización precoz.

Con pequeñas diferencias (dejando las agujas fuera o subcutáneas y con yeso o sin él) se han publicado muy buenos resultados con este método, casi siempre en fracturas extraarticulares. Goyeneche y Torre $^{33}$ revisaron 128 pacientes obteniendo resultados excelentes y buenos por encima del $85 \%$ de los casos.

\section{VIII.2.4. Fijación elástica:}

En 1969, el francés Py ${ }^{34}$ dio a conocer su método de enclavado isoelástico, introduciendo dos agujas, una por la estiloides radial y otra por la cara posterior de la columna intermedia, que no toman la segunda cortical sino que discurren sobre ella por el canal medular a veces hasta el 1/3 proximal del hueso haciendo un efecto de ballesta (Figura 5). Resultó muy bueno para las fracturas con el fragmento distal desplazado dorsalmente, con o sin conminución, y cuando existe un fragmento postero-interno, pero no resuelve las fracturas multifragmentarias.

Basado en este mismo principio, Sato et al. ${ }^{35}$ comunicaron buenos resultados en 29 pacientes introduciendo las agujas en sentido distal por dos orificios efectuados en la cara dorsal del radio a unos 7-10 $\mathrm{cm}$ proximales a la estiloides radial.

\section{VIII.2.5. Tracción bipolar con agujas de Kirsch- ner:}

El método de tracción bipolar con Kirschner (TBK), indicado en fracturas articulares o muy conminutas en las que no se pueda conseguir la estabilidad sólo con escayola ni con fijación percutánea de los fragmentos, consiste en introducir dos agujas transóseas, una proximal y otra distal al foco de fractura, hacer la reducción y, manteniendo la tracción, poner una escayola en la que quedan incluidas las agujas.

Está basado en el criterio de que la tracción de los ligamentos extrínsecos de la muñeca unidos a los fragmentos óseos, íntegros aun cuando exista gran conminución, ayuda a la reducción anatómica de la fractura y su tensión mantenida evita el acortamiento radial. Este principio, descrito posteriormente por Jacques Vidal ${ }^{36}$ en 1977 con el nombre de ligamentotaxis, es el que justifica el uso de los fijadores externos. 

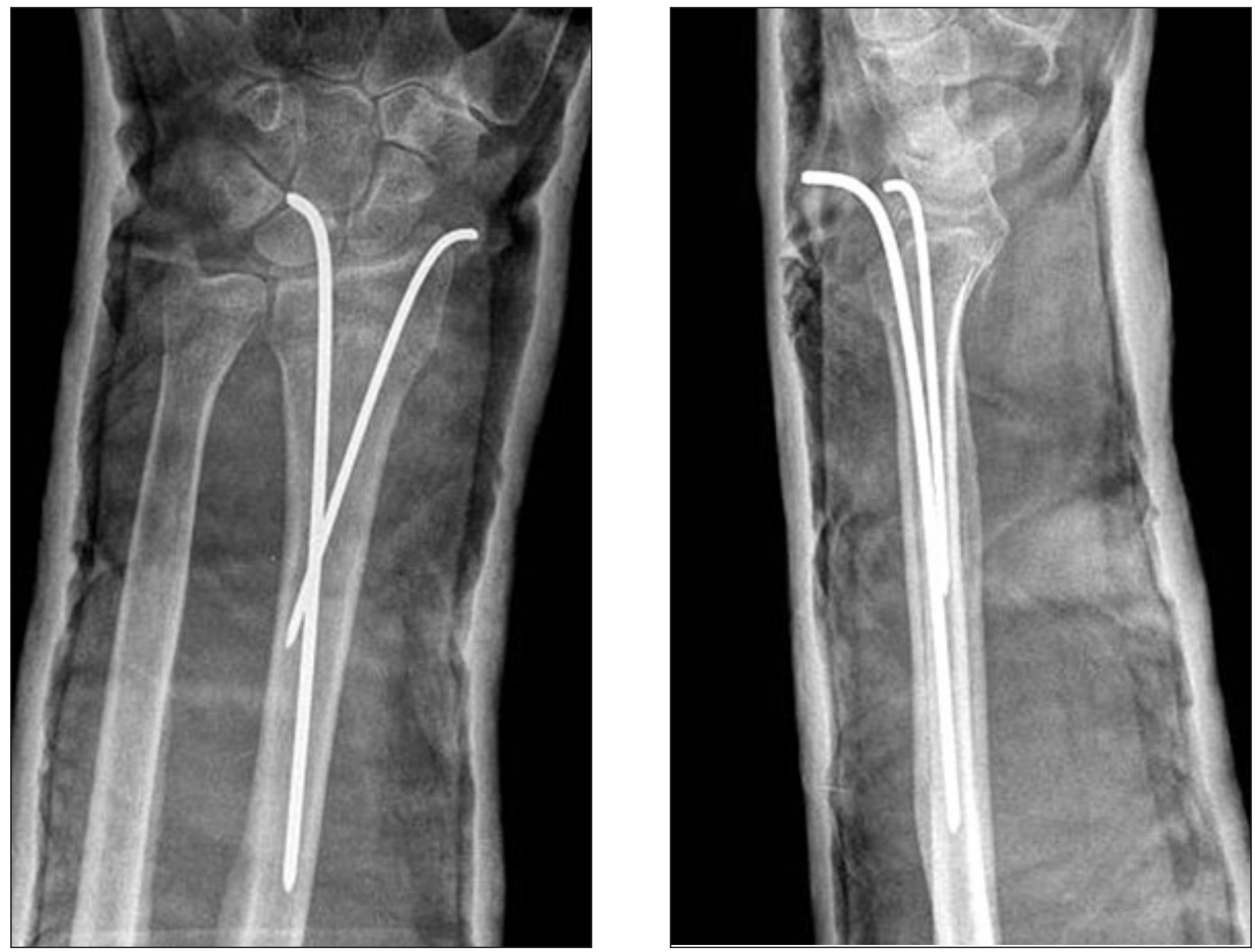

Figura 5: A y B: Proyecciones lateral y AP de una fractura A2 tratada con el método de Py ${ }^{34}$.

A la técnica original de TBK, descrita por Böhler en $1929^{37}$, con una aguja por la base de los últimos cuatro metacarpianos y otra en el tercio proximal del cúbito, le siguieron numerosas variantes que tomaban sólo el primer metacarpiano (método de Ledoux et al. ${ }^{38}$ ) o el segundo y tercero en la zona distal y el cúbito y radio conjuntos o individualmente en la proximal (Figura 6). Algunos autores prefieren el uso de yesos braquio-palmares y otros dejan libre el codo. En cualquier caso, para que el yeso mantenga la tracción deben emplearse agujas gruesas (de 2 ó $2,5 \mathrm{~mm}$ en los metacarpianos y de 2,5 o $3 \mathrm{~mm}$ en el antebrazo) y mantenerse un tiempo de 8 semanas, lo que aumenta la probabilidad de rigidez.

Desde la aparición de los fijadores externos este método está un poco en desuso pero fue muy empleado en la segunda mitad del siglo pasado. Surer et al. ${ }^{39}$ y Dujardin et al. ${ }^{40}$ obtuvieron resultados buenos y muy buenos en más del $74 \%$ de los casos en 80 y 56 pacientes, respectivamen- te, con el método de Ledoux et al. ${ }^{38}$. En nuestro medio, Irisarri y Alcocer ${ }^{41}$ publicaron buenos resultados en 16 pacientes tratados con una aguja proximal que tomaba cúbito y radio, y una distal por la base de los cuatro últimos metacarpianos.

\section{VIII.2.6. Complicaciones del tratamiento percu- táneo:}

Las complicaciones de la fijación percutánea son frecuentes y pueden llegar a ser graves. Son necesarias unas estrictas medidas de asepsia y un buen conocimiento anatómico de la zona para evitar la lesión de los nervios, vasos y tendones que cruzan la muñeca. La cara anterior se debe evitar por la presencia de las arterias radial, cubital, el nervio mediano y los tendones flexores. En la posterior, las punciones tendinosas pueden causar adherencias y rigidez. La fijación por la apófisis estiloides del radio puede lesionar el nervio radial y las fijaciones desde el borde interno al nervio cubital. 


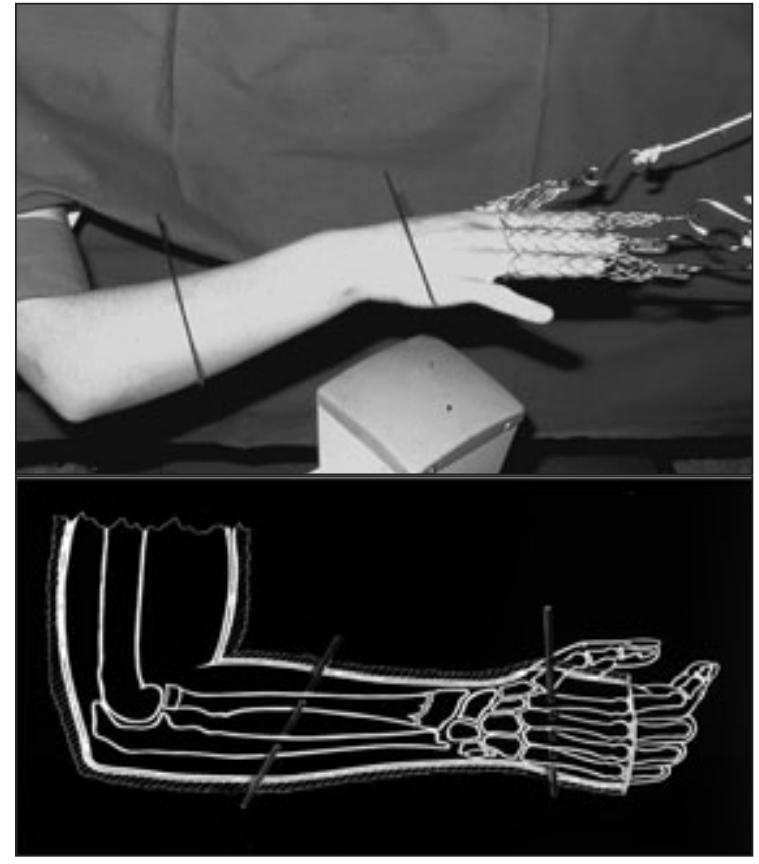

Figura 6: A: Variante del método de Böhler ${ }^{37}$. B: Dibujo cortesía del Dr. JL Haro.

Las agujas pueden doblarse, aflojarse y migrar en sentido distal o proximal. Dejarlas subcutáneas obliga a su retirada con anestesia local; extracutáneas causan reacción inflamatoria o infección cutánea en el 30\% de los casos y aumenta el riesgo de osteomielitis.

\section{VIII.3 Fijación interna:}

De forma general, se elige la reducción abierta para las fracturas articulares en pacientes activos con calidad ósea aceptable y sin patología concomitante de interés en los que no se puede conseguir una restauración de las superficies articulares por medios cerrados de manipulación, ligamentotaxis o fijación percutánea, y cuando hubo un desplazamiento significativo tras una reducción inicial. Otros criterios importantes son la complejidad de una lesión articular, conminución metafisaria, inestabilidad, asociación con otras lesiones del carpo, presencia de fracturas ipsilaterales concomitantes y/o luxaciones en el miembro superior.

Actualmente, por la aparición en el mercado de los nuevos sistemas de fijación con gran soporte subcondral que brindan un efecto de fijador externo pero de colocación interna, esto no es tan concluyente y cada vez se emplean más las placas en personas de avanzada edad, incluso en fracturas articulares ${ }^{42,43}$, lo que acelera la rehabilitación y disminuye las complicaciones de la inmovilización prolongada.

La fijación interna se realiza con tornillos interfragmentarios cuando hay grandes fragmentos desplazados (fractura de la estiloides radial, tipo B1, por ejemplo) o con placas en las fracturas metafiso-epifisarias. Las placas proporcionan una transferencia de cargas de la superficie articular a la diáfisis ponteando la zona metafisaria y restablecen la inclinación volar del radio.

Los factores que limitan la reconstrucción con placas o tornillos interfragmentarios son el número de fragmentos (más de 4 ó 5), tamaño de los mismos, cantidad y calidad del hueso esponjoso y lesiones asociadas de cartílago. No deben emplearse durante el período de crecimiento y cuando haya infección o insuficiente cobertura de partes blandas.

\section{VIII.3.1. Técnicas y abordajes:}

La elección de la vía de abordaje depende de la localización y dirección de desplazamiento de los fragmentos, pero desde la aparición de las nuevas placas volares, se está prefiriendo esta vía, que en buenas manos permite reducir ciertas fracturas dorsales y resolver fracturas combinadas de forma satisfactoria.

\section{VIII.3.1.1. Reducción abierta amplia:}

a) La vía volar clásica que da acceso a la zona metafiso-epifisaria distal del radio en su cara volar, con la incisión cutánea sobre el flexor carpi radialis, fue recientemente mejorada por Orbay et al. ${ }^{44,45}$ con un ángulo en la zona distal, sobre el pliegue proximal de flexión volar de la muñeca, y la sección del brachioradialis, lo que permite pronar el fragmento proximal del radio para acceder a la zona dorsal de la fractura.

Está indicada en las fracturas metafisarias y articulares no reductibles, en las marginales anteriores (tipo B3 de la AO), en luxaciones radiocarpianas, cuando hay pérdida precoz de la reducción, asociación de lesiones del carpo, de los nervios o vasos volares y otras fracturas del antebrazo o codo ipsilaterales.

Las nuevas placas volares de bajo perfil, con tornillos bloqueados a la placa y pernos subcondrales aportan innumerables ventajas: pueden 
colocarse lo más distal posible sin que sea necesario abrir el túnel carpiano, evitan atravesar la cortical dorsal para no dañar los tendones extensores, por la versatilidad de inclinación de sus tornillos permiten fijar fragmentos que no se lograría con las antiguas placas y pueden tratarse con ellas fracturas desplazadas dorsalmente ${ }^{44,45}$.

Una vez colocadas debe verificarse siempre con el intensificador de imágenes que se restauró la congruencia articular, se restableció la longitud e inclinaciones anatómicas del radio y que los tornillos no sobresalen excesivamente en la cara dorsal ni penetran en la articulación. Para comprobar este último aspecto debe hacerse la visión lateral con el codo apoyado en la mesa y la muñeca levantada entre 20 y $25^{\circ}$ para que el haz radiológico sea paralelo a la glena radial.

b) Vía dorsal: Como la mayoría de las FEDR tienen conminución dorsal y algunas fracturas articulares con desplazamiento posterior son muy difíciles de reducir por vía anterior, el abordaje dorsal, con incisión longitudinal sobre el tubérculo de Lister, proporciona un buen campo de visión de toda la cara posterior del radio y facilita la reducción y fijación de la fractura. Pero existen claras diferencias anatómicas entre las caras volar y dorsal del radio que condicionan el implante de placas sobre sus superficies. La cara volar, ocupada por el pronator quadratus, es cóncava y está alejada de los tendones flexores, mientras que la superficie dorsal del radio, convexa, está en íntimo contacto con los tendones extensores, por lo que las placas, aún de bajo perfil (tipo $\mathrm{Pi}^{\circledR}$, de $\mathrm{AO}$ ) pueden producir adherencias, tenosinovitis y roturas tendinosas; de ahí que sea recomendable su extracción una vez consolidada la fractura, si bien estas complicaciones pueden minimizarse interponiendo una banda del ligamento anular posterior del carpo entre la placa y los tendones extensores ${ }^{46}$.

VIII.3.1.2. Reducción abierta a través de abordajes mínimos: La extremidad distal del radio no escapó a la tendencia actual de la cirugía mínimamente invasiva y se ha publicado la colocación de placas volares a través de dos cortas incisiones en lugar de una larga ${ }^{47}$. Al margen de estas modas, los pequeños abordajes están indicados cuando existen fragmentos desplazados de escaso tamaño. El lugar de incisión depende de la localización del fragmento. Si es volar y cubital es preferible la vía anterior; si es dorso-cubital se accede mejor por una vía dorsal a través del quinto compartimento extensor.

VIII.3.1.3. Reducción artroscópica: Fue Whipple $^{48}$, en 1995, el primero en emplear la artroscopia como método de reducción y fijación de las FEDR. Las ventajas de esta técnica son la correcta reducción de fragmentos articulares, la extracción de pequeños fragmentos osteocondrales irreductibles y la valoración de las lesiones ligamentosas asociadas ${ }^{49}$.

\section{VIII.4 Fijación externa:}

La fijación externa, como la TBK, se fundamenta casi siempre en el intento de mantener la tracción para evitar el colapso de los fragmentos. En la muñeca se utiliza desde hace más de 80 años, introducida por Ombrédanne para tratar las fracturas distales del radio en niños y adolescentes $^{50}$.

A pesar del actual auge de la fijación interna con placa palmar, los fijadores externos tienen aun hoy ciertas indicaciones de uso preferente:

- En fracturas abiertas con amplia exposición o pérdida de tejidos blandos.

- En fracturas extraarticulares irreducibles o muy inestables por importante conminución metafisaria o gran pérdida de sustancia ósea por impactación.

- En fracturas intraarticulares complejas, a veces en combinación con fijación interna.

- Como medida temporal en pacientes politraumatizados o a la espera de transferir al paciente a un centro de referencia para el tratamiento definitivo.

- En algunos casos, tras reducción provisional previa a TAC para comprender bien el trazo de fractura, después de desplazamientos secundarios o como método de fijación en el tratamiento de consolidaciones viciosas.

Las contraindicaciones son la inestabilidad de la articulación radiocubital distal, fractura de Barton o Barton inversa, rotura de los ligamentos radiocarpianos volares (luxación radio-carpiana) y la conminución metafisaria. 
Al decidirse por este método de tratamiento hay que tener en cuenta el curso de los tendones y nervios en la zona de implante de los tornillos y una serie de consideraciones mecánicas:

a) Cualquier implante que se use debe ser lo suficientemente fuerte para neutralizar las cargas fisiológicas que atraviesan la muñeca, definidas por Rikli et al. ${ }^{51}$ en dos centros de transmisión de fuerzas (columnas radial e intermedia).

b) La fijación externa es considerada como un implante flexible ${ }^{52}$.

c) La solidez del fijador depende de la rigidez de las barras conectoras y las abrazaderas, cuya consistencia puede aumentarse aumentando su diámetro. En los fijadores uniplanares, que son los más comunes, se aumenta la rigidez colocando 2 barras conectoras paralelas. La colocación cercana a la piel de las barras disminuye el brazo de palanca y por consiguiente las fuerzas de cizallamiento. También le aporta mayor rigidez la mejor colocación de los clavos: la fijación al hueso mejora taladrando un milímetro menos del diámetro del clavo y con la toma bicortical. La colocación de los clavos cercanos al foco de fractura disminuye el brazo de palanca y por tanto las fuerzas de cizallamiento, pero su distribución por la diáfisis distribuye la fuerza por toda la longitud de la misma, por lo que lo ideal es insertar un clavo cerca del foco de fractura y el segundo lo más lejos posible. Aumentar la rigidez del marco no aumenta la rigidez interfragmentaria.

Después de restablecer la longitud y la inclinación radial se puede aumentar la rigidez de la fijación de varias maneras: colocando un clavo que bloquee la estiloides, añadiendo un $5^{\circ}$ clavo en una expansión del propio fijador, colocando un clavo dorsal que evite la inclinación dorsal del fragmento distal, aumentando la fijación con agujas de Kirschner que atraviesen el foco, o bien añadiendo al marco del fijador agujas de Kirschner sin atravesar el foco.

\section{VIII.4.1 Fijadores externos transarticulares (en puente):}

La obtención de la reducción y su mantenimiento recaen en el principio de la ligamentotaxis $^{36}$. La tracción longitudinal sobre el carpo se transmite a los ligamentos radio-semilunar largo y radio-escafo-hueso grande y restablece la al- tura radial; de manera similar, la pronación del carpo reduce la supinación del fragmento distal. Pero la ligamentotaxis tiene unos límites ya que los ligamentos tienen un comportamiento viscoelástico y se relajan tras un período de tracción. Esta tracción no corrige en sí misma la inclinación dorsal e incluso, si es excesiva, puede aumentarla porque los ligamentos radio-carpianos volares son más gruesos y cortos que los dorsales. Por otra parte, en las fracturas intraarticulares la tracción puede conseguir la reducción de la estiloides radial pero no de la fosa semilunar impactada ni del fragmento volar medial, en el que aumenta la rotación. Además, con estos fijadores no se controla el desplazamiento radial del fragmento distal, por lo que no deben usarse en fracturas con inestabilidad radio-cubital.

Las complicaciones más frecuentes son la movilización del fijador (puede prevenirse con revisión periódica y reajuste de las conexiones), complicaciones de los clavos (aflojamiento, infección, interferencia con el aparato extensor, riesgo de lesión nerviosa) y el exceso de distracción, tanto en grado como en tiempo, que puede causar rigidez digital por el aumento de fuerza requerida para la flexión de las metacarpo-falángicas y de la muñeca por contractura en pronación; al tiempo que se aumenta la presión en el canal del carpo.

Se han descrito en la literatura ${ }^{52,53}$ una tasa de buenos resultados similar a la reducción abierta y fijación interna en la mayoría de los pacientes con el uso de fijación externa siempre que se evite el exceso de distracción, de flexión y pronación y la duración del tratamiento tenga una media de 7 semanas $^{54}$.

\section{VIII.4.2 Fijadores externos articulados (sin puente):}

En un esfuerzo de permitir el movimiento precoz de la muñeca se han desarrollado muchas expansiones de los fijadores transarticulares (Pennig ${ }^{\circledR}$, Dynawrist ${ }^{\circledR}$, etc.) pero a menudo fracasan porque no pueden reproducir la compleja dinámica del carpo. Los fijadores articulados permiten una movilización precoz por lo que los resultados en movilidad y fuerza suelen ser mejores. Sus indicaciones incluyen fracturas extraarticulares inestables y fracturas intrarticulares en 2 y 3 partes con buena calidad ósea y 
articulación radiocubital estable. Están contraindicados en las fracturas marginales anterior o posterior y en niños con fisis abiertas. En las fracturas con conminución metafisaria o diafisaria se requiere a menudo osteosíntesis complementaria.

Las complicaciones son debidas a la movilización de los clavos por fragmentos muy pequeños, hueso de mala calidad, reducción demasiado vigorosa, fallos en la reducción, lesión nerviosa, interferencia con el aparato extensor y colapso tardío de la fractura, que puede aparecer incluso 6 meses después de la misma en hueso osteoporótico ${ }^{55}$ En la literatura se encuentran buenos resultados con el uso de fijadores articulados tanto en las fracturas extraarticulares como en las articulares con escalón residual menor de $2 \mathrm{~mm}^{56,57}$.

\section{VIII.5 Fijación combinada:}

Como ya se ha dicho, ningún método es óptimo para reducir y fijar todos los posibles desplazamientos. Con frecuencia, algunas fracturas conminutas y articulares suponen un reto a la pericia del cirujano y requieren la combinación de dos o más sistemas de osteosíntesis en la búsqueda de una reducción lo más anatómica posible. Caben y valen todas las combinaciones: placa y agujas (Figura 7a), fijador externo más agujas (Figura 7b), fijador externo más placa y agujas (Figura 8c), fijador externo más agujas intrafocales (Kapandji) y fijación cubital (Figuras 7 d, e, f), etc.

Además de la osteosíntesis metálica, cuando hay una notoria pérdida de masa ósea por conminución o impactación a veces es necesario rellenar el defecto con hueso autólogo, cemento óseo o espaciadores de hidroxiapatita ${ }^{58}$.

\section{COMPLICACIONES}

Las FEDR tienen una gran morbilidad; del 20 al 50\% tienen malos resultados ${ }^{59}$ y aun cuando algunas secuelas son muy bien toleradas por el paciente, otras pueden llegar a hacer imposibles las simples actividades de la vida diaria. Las complicaciones, precoces o tardías, más habituales son las siguientes:

\section{IX.1. Desplazamiento secundario de las fracturas inestables:}

Es preciso enfatizar la importancia de verificar radiológicamente la estabilidad de las fracturas que han sido manipuladas, entre el $5^{\circ}$ y el $7^{\circ}$ días post-reducción. McQueen et al. ${ }^{60}$ estudiaron a 120 pacientes con desplazamientos secundarios dividiéndolos en grupos de 30 pacientes a los que trataron con cuatro métodos diferentes: remanipulación y yeso, reducción abierta más injerto óseo, fijación externa con movilización en la $3^{a}$ semana, y fijación externa sin movilización en la $3^{\mathrm{a}}$ semana. No encontraron diferencias significativas en la funcionalidad al cabo de un año, aunque sí en la restauración anatómica: los mejores resultados se obtuvieron con la reducción abierta más injerto y los peores con la remanipulación y yeso.

Jupiter et al. ${ }^{42}$ trataron con placas (12 volares, 8 dorsales) a 20 pacientes con fracturas desplazadas secundariamente obteniendo un $95 \%$ de buenos y excelentes resultados.

En consecuencia, en los desplazamientos secundarios, especialmente en mujeres osteoporóticas, parece preferible la fijación interna a la remanipulación.

\section{IX.2. Consolidación defectuosa, rigidez y dolor:}

Éstas son, sin duda, las complicaciones más frecuentes. La consolidación defectuosa ocurre bien porque no se logró una reducción perfecta, bien porque ésta se haya perdido. Es habitual en las fracturas que no han sido tratadas, cuando se emplearon yesos flojos o mal conformados, y tras osteosíntesis mal indicadas (agujas en fracturas muy inestables) o mal ejecutadas.

Los desplazamientos más frecuentes son el acortamiento radial y la inclinación dorsal de la carilla del radio. En el primer caso suele haber una luxación o subluxación radiocubital distal, lo que produce un defecto estético debido a la prominencia de la cabeza cubital y limitación dolorosa de la pronosupinación e inclinaciones laterales. Con frecuencia, el cúbito llega a contactar con los huesos carpianos produciendo un síndrome de impactación cubital (Figura 8). El tratamiento, valorando siempre el grado de desplazamiento, edad del paciente, actividad, etc., 

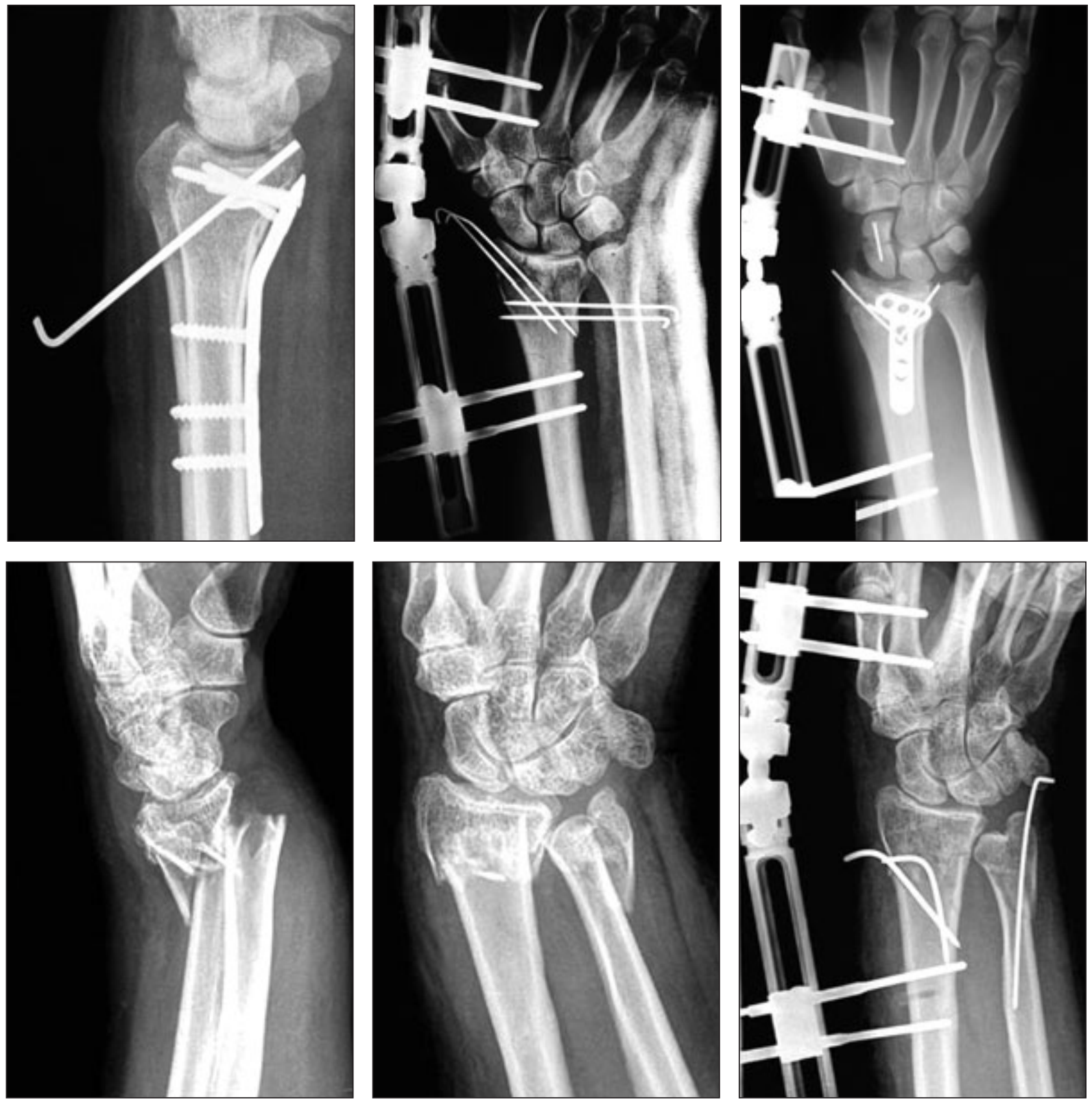

Figura 7: Tratamiento combinado. A: La placa no llega a sujetar un fragmento largo y delgado del labio anterior de la epífisis, fijado con una aguja que se extraerá por el dorso a partir de la $3^{a}$ semana. B: Fractura tipo C3 tratada con fijador externo, agujas de Kirschner y férula temporal de escayola. C: (Cortesía del Dr. S Pombo): Fractura articular y conminuta tratada con fijador externo, placa y agujas, asociada a fractura del escafoides. $D, E, F$ : fijador externo más agujas intrafocales (Kapandji) y fijación cubital.

consistiría en la escisión de la cabeza cubital (técnica de Darrach o variantes) o en una resección del cuello cubital con artrodesis radio-cubital distal (técnica de Sauvé-Kapandji).

En el segundo caso, cuando la angulación dorsal de la carilla radial produce dolor y limita mucho la flexión, el tratamiento consiste en una osteotomía radial correctora con aporte de hueso esponjoso. Puede realizarse por vía anterior fi- jando el foco con una placa, pero la vía posterior ofrece más comodidad para intercalar en el foco de la osteotomía un injerto trapezoidal obtenido de la cresta ilíaca. También en este caso la fijación puede efectuarse con placa, aunque los autores de este trabajo solemos realizar la osteosíntesis con dos simples agujas de Kirschner para evitar las posibles lesiones de los tendones extensores (Figura 9). 

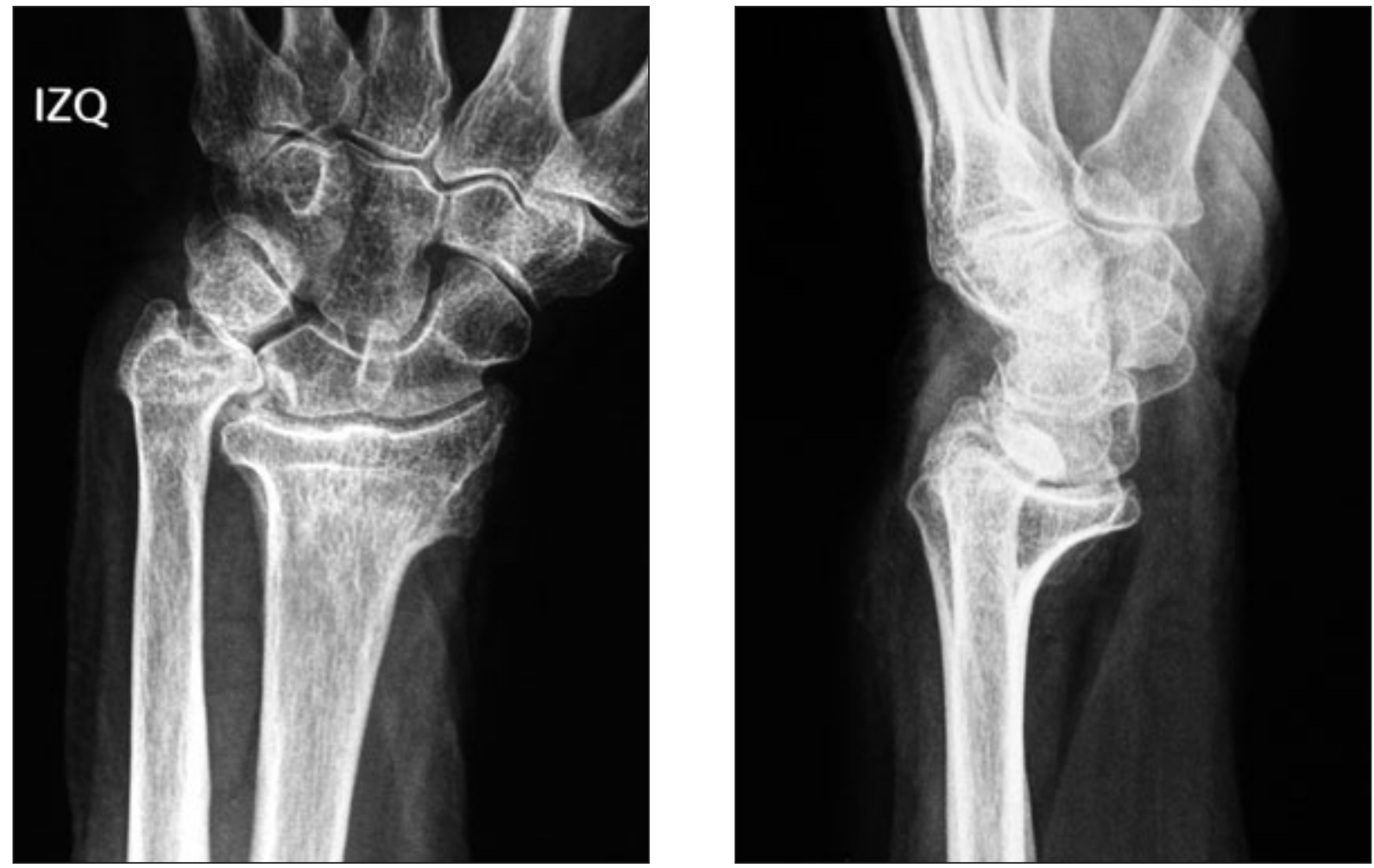

Figura 8: A y B: Proyecciones AP y lateral de un síndrome de impactación cubital por acortamiento del radio.
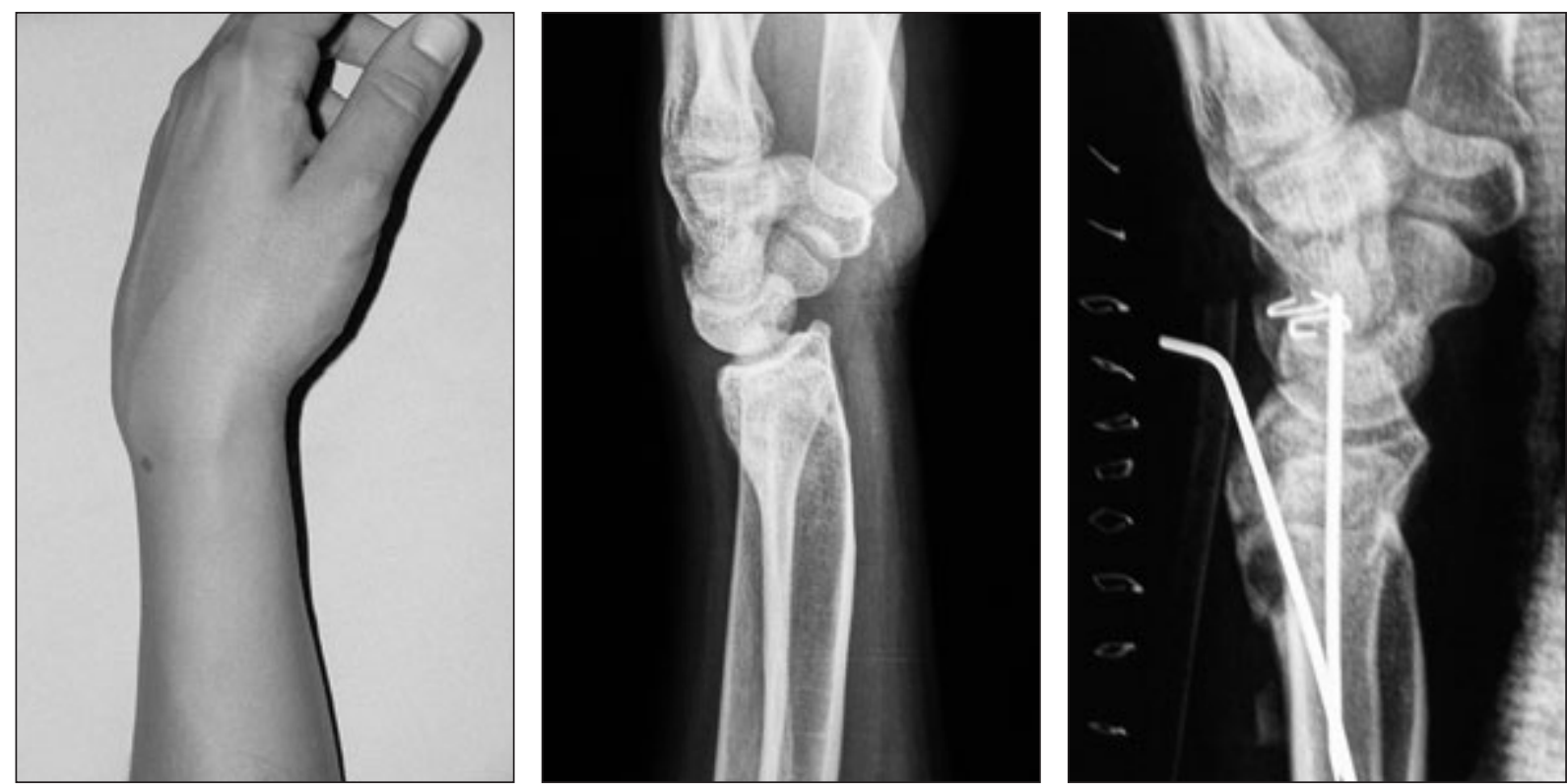

Figura 9: A: Aspecto clínico de un paciente de 22 años que había fracturado tres veces la misma muñeca. B: desviación dorsal de la carilla radial en $44^{\circ}$. C: Osteotomía correctora; apréciese el injerto triangular en el dorso.

La osteotomía correctora de pequeños fragmentos articulares mal consolidados puede realizarse mediante la visualización con artroscopia ${ }^{61}$, técnica difícil pero que en manos experimentadas ofrece reducciones muy precisas.
Si la consolidación defectuosa es de larga evolución y existe artrosis radio-carpiana o mediocarpiana, se puede, en algún caso, recurrir a una artrodesis. No obstante, aun siendo tan frecuentes las consolidaciones viciosas, no siempre 
necesitan tratamiento ya que para las actividades propias de la mujer añosa no se requiere toda la movilidad de la muñeca, por lo que antes de indicar un tratamiento quirúrgico reparador debemos valorar mucho más los aspectos clínicos (dolor, edad, actividad) que los radiológicos.

\section{IX.3. Retardo de consolidación y seudoartrosis:}

La seudoartrosis es muy rara incluso en pacientes de avanzada edad y con osteoporosis. El tratamiento con fijadores externos o con tracción bipolar con agujas de Kirschner puede causar retardos de consolidación por la excesiva distracción en el foco de fractura. Aunque la mayor parte de las fracturas suelen consolidar inmovilizándolas el tiempo suficiente, es mayor el peligro de rigidez por inmovilizaciones prolongadas que el de seudoartrosis, por lo que se deben evitar los tiempos excesivos de enyesado.

\section{IX.4. Síndrome doloroso regional complejo:}

Es muy frecuente, sobre todo con el empleo de fijadores externos tal vez por la excesiva trac-

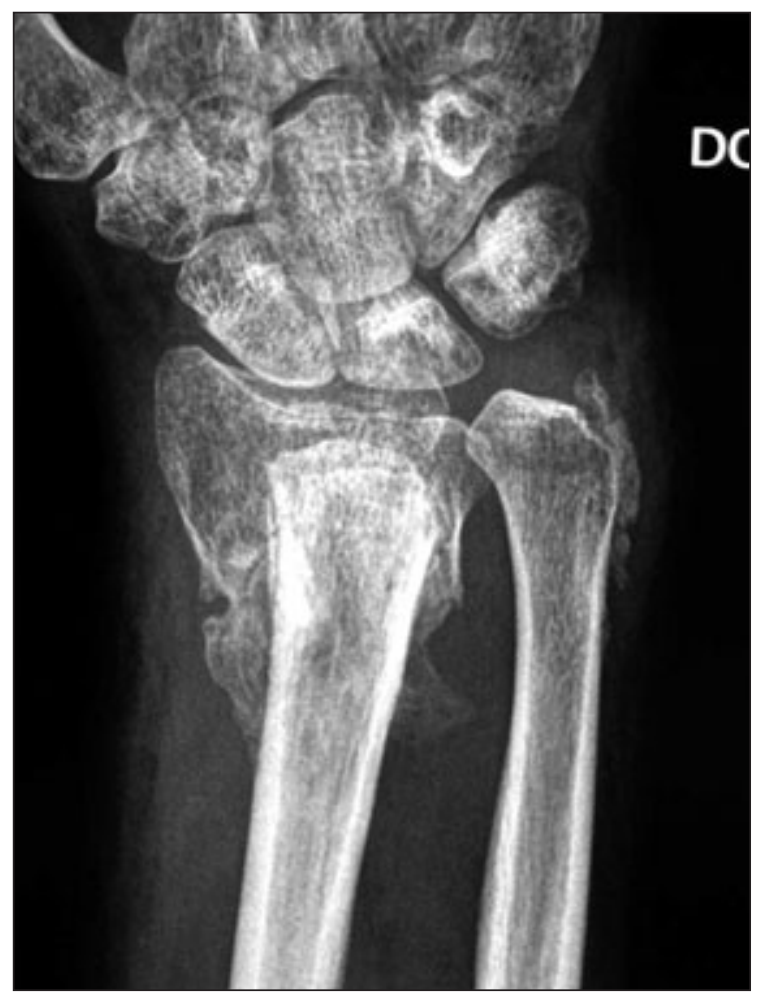

ción de los vasos. Se caracteriza por osteopenia, dolor, rigidez de muñeca y dedos, edema, piel lisa, brillante y sudorosa. El tratamiento del proceso ya establecido es arduo, por lo que es fundamental su prevención con analgésicos y medidas antiedema para realizar una movilización precoz.

\section{IX.5. Síndrome compresivo del nervio mediano:}

La compresión del nervio mediano puede ser precoz o tardía. El síndrome del túnel carpiano (STC) agudo se produce por la tumefacción, el hematoma fracturario, la compresión por algún fragmento anterior o exageradas posturas de inmovilización. Deben evitarse las posturas en desviación cubital y flexión forzadas por esta razón.

El STC tardío es raro porque, como bien explica Lluch ${ }^{62}$, estas fracturas no producen una deformidad en la luz del túnel sino a un nivel más proximal. En una revisión efectuada por uno de los autores ${ }^{63}$ sobre el STC de origen secundario, de 317 muñecas operadas sólo 7 casos fueron debidos a FEDR (2\%).

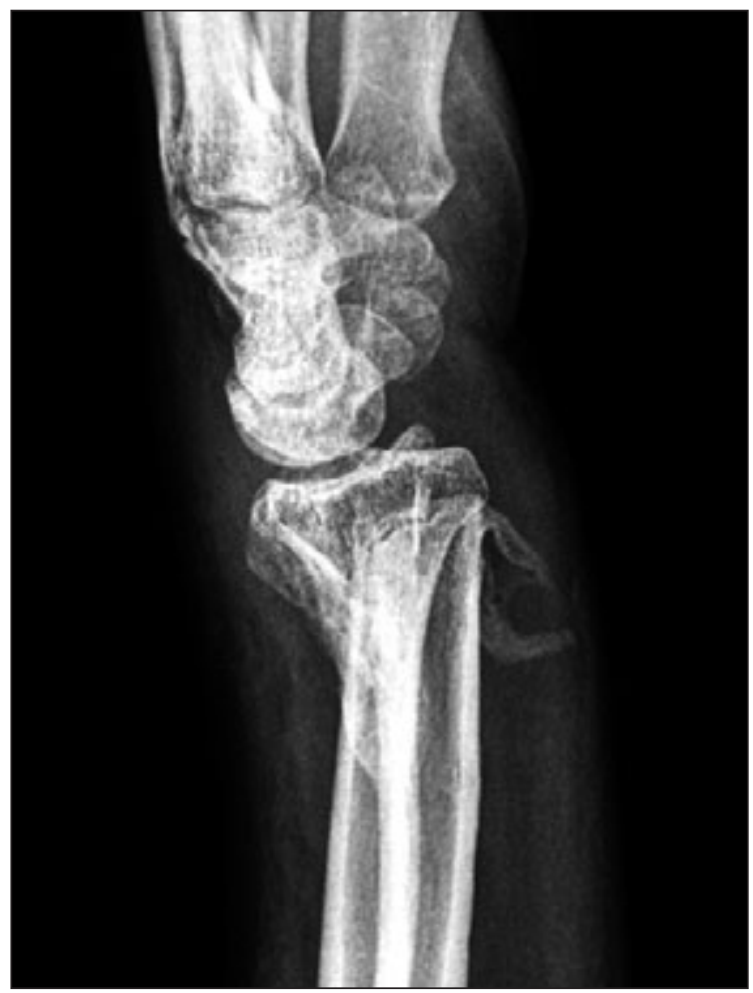

Figura 10: A: Consolidación defectuosa en un paciente que ño recibió tratamiento debido a la concomitancia de un grave politraumatismo. B: En la proyección lateral se aprécia un callo hipertrófico volar que causaba un STC. 
No se produce un aumento de presión en el túnel carpiano tras la colocación de placas volares, por lo que no es necesario abrir el retináculo cuando se emplea este método de osteosíntesis.

En algunas ocasiones la compresión nerviosa puede originarse en el foco de fractura por calcificaciones o callos hipertróficos, especialmente en fracturas que no fueron tratadas. La Figura 10 muestra la muñeca de un paciente que fue ingresado en la UCI tras un politraumatismo y su fractura no recibió tratamiento debido al grave estado general.

\section{IX.6. Lesiones tendinosas:}

La tenosinovitis dolorosa de los flexores suele deberse al roce de los tendones con la arista fracturaria o con el material de osteosíntesis. La tenosinovitis de los extensores es frecuente tras el implante de placas dorsales y la rotura se produce a veces por la fricción de agujas subcutáneas.

La rotura del extensor pollicis longus es infrecuente; Meine ${ }^{64}$ encontró 8 roturas en 594 fracturas revisadas $(1,5 \%)$. Suele ocurrir tardíamente, a partir de la $4^{\mathrm{a}}$ semana y a veces cuando ya la paciente está sin yeso. Se debe al roce del tendón con el foco de fractura o con el tubérculo de Lister. En pacientes ancianos quizás no se necesite tratamiento ya que la secuela es discreta y a menudo hay una recuperación espontánea de la extensión activa. En pacientes jóvenes con incapacidad manifiesta se debe hacer una transposición del extensor indicis proprius.

\section{IX.7. Infecciones:}

Las infecciones óseas son muy raras. Ocurren en las fracturas expuestas o en las que han sido tratadas quirúrgicamente. En cambio son muy frecuentes las infecciones cutáneas cuando se emplean fijadores externos o agujas transcutáneas.

\section{IX.8. Otras:}

Otras complicaciones descritas en las FEDR, sumamente raras pero descritas en la literatura, son el síndrome hombro-mano ${ }^{64}$, epicondilitis ${ }^{64}$, síndrome compartimental, lesiones vasculares o sinostosis radio-cubital distal.

\section{LESIONES ASOCIADAS}

Si exceptuamos la asociación con la fractura de apófisis estiloides cubital, la mayoría de las FEDR suelen presentarse como lesiones aisladas. Pero cuando el mecanismo lesional es de cierta energía, estas fracturas no son infrecuentes en los politraumatizados y en el miembro superior pueden asociarse con fracturas de los otros huesos del carpo, lesiones ligamentosas, de partes blandas y de la membrana interósea del antebrazo $^{65,66}$.

\section{FRACTURAS DE LA EXTREMIDAD DISTAL DEL CÚBITO}

La cabeza del cúbito es el punto fijo sobre el que gira la epífisis distal del radio en los movimientos de pronosupinación, y la importancia de sus lesiones no siempre está reconocida ${ }^{67}$ a pesar de que con mucha frecuencia las FEDR desplazadas se asocian a lesiones en el borde cubital de la muñeca: fracturas del cúbito (apófisis estiloides, cabeza o diáfisis) y roturas del complejo fibrocartílago triangular.

\section{XI.1. Clasificación}

Ring et al. ${ }^{68}$ modificaron la clasificación de la AO sobre las fracturas de la extremidad distal del cúbito estableciendo una división en 6 tipos:

Q1: Fractura de la base de la estiloides cubital

Q2: Fractura simple del cuello

Q3: Fractura conminuta del cuello

Q4: Fractura de la cabeza

Q5: Fractura de cabeza y cuello

Q6: Fractura proximal al cuello

\section{XI.2. Tratamiento}

El tratamiento de estas lesiones está influido por el tratamiento del radio distal. En la toma de decisiones debemos buscar en primer lugar la reducción anatómica de la fractura del radio y posteriormente valorar la estabilidad de la articulación radiocubital distal (RCD) y el desplazamiento de la fractura cubital ${ }^{69}$. La estabilidad de la RCD depende de unos estabilizadores está- 
ticos (congruencia ósea entre la cabeza cubital y la fosa sigmoidea del radio) y otros dinámicos, fundamentalmente el complejo fibrocartílago triangular, cuya parte periférica la forman los ligamentos radio-cubitales que discurren desde la base de la estiloides y la fóvea cubitales hasta el borde de la fosa sigmoidea ${ }^{70}$.

En general, si tras la reducción de la FEDR, la RCD es estable o parcialmente estable se trata con inmovilización durante 4-6 semanas en la posición de reducción. En las inestables e irreductibles si hay fractura desplazada de la estiloides cubital debe hacerse reducción abierta más fijación interna, y si no hay fractura debe repararse el complejo fibrocartílago triangular y fijar la RCD con agujas de Kirschner durante 4-6 semanas.

\section{XI.2.1 Fractura de la apófisis estiloides cubital (Q1):}

Una gran parte de las fracturas de la estiloides cubital se reducen al reducir la fractura de radio. Cuando hay una pequeña fractura de la punta de la estiloides se presupone que no hay desinserción ligamentaria y se puede tratar mediante yeso. Si la fractura de la base de la apófisis está desplazada más de $2 \mathrm{~mm}$, lo que implica la rotura de los ligamentos radio-cubitales, se recomienda reducción y osteosíntesis abierta. Las opciones serían osteosíntesis con agujas de Kirschner, banda de tensión u osteosíntesis con tornillo.

Si el fragmento fuera insuficiente para la osteosíntesis puede realizarse un reanclaje del ligamento en la fóvea de la cabeza cubital extirpando el fragmento óseo.

En el caso de que hayamos realizado la estabilización de la fractura radial mediante fijación externa hay accesorios que bloquean la pronosupinación y se pueden añadir al marco para evitar el abordaje directo de la fractura.

La seudoartrosis de la fractura no ha demostrado empeorar la evolución clínica de los pacientes respecto a aquéllos en los que sí se ha conseguido la consolidación ${ }^{71-72}$.

\section{XI.2.2. Fracturas del cuello y diafisarias distales} (Q2, Q3 y Q6):

Las fractura del cuello y las fracturas diafisarias distales del cúbito simples suelen ser estables tras la reducción de la fractura radial. En este caso no se recomienda tratamiento quirúrgico y es suficiente con la inmovilización con un yeso braquio-palmar.

Cuando la fractura es irreductible o inestable se debe realizar reducción abierta y osteosíntesis mediante clavo-placa, placa a compresión o banda a tensión suplementada por tornillos interfragmentarios ${ }^{68}$.

El clavo placa puede ser de 2, 2,4 ó 2,7 mm como los usados habitualmente en las fracturas de metacarpianos y falanges. Los resultados que presentan son muy satisfactorios ${ }^{68}$. Las placas a compresión de $3,5 \mathrm{~mm}$ son demasiado voluminosas para una zona tan superficial $\mathrm{y}$ tienen pocos orificios distales, por lo que se han desarrollado placas con gancho o moldeadas y con estabilidad angular que permiten tornillos de $2 \mathrm{~mm}$.

\section{XI.2.3. Fracturas de la cabeza cubital (Q4, Q5):}

En las fracturas intrarticulares y conminutas de la cabeza cubital se recomienda reducción de la alineación del cúbito y de la articulación mediante manipulación y yeso braquio-palmar. Si no fuera posible se debe realizar reducción abierta y osteosíntesis con tornillos tipo Herbert, agujas de Kirschner u Orthosorb para la fractura de la cabeza, y una placa condílea bloqueada o banda de tensión para la fractura extraarticular, manteniendo además bloqueada la articulación con yeso o con fijador externo.

Otra alternativa sería no tratar esa fractura en el momento agudo y realizar procedimientos de salvamento en la fase de secuelas.

Los procedimientos de salvamento más agresivos, como la sustitución protésica o la resección e interposición, no deben considerarse como alternativa inicial ${ }^{73}$ sino reservarse para la fase de secuelas. Entre ellos debemos incluir la resección tipo Darrach y variantes o la artroplastia de Sauvé- Kapandji. En los últimos años se ha propuesto tratar las lesiones de la articulación radiocubital distal con sustitución protésica, bien mediante hemiartroplastia tipo Herbert o Eclipse, y si ambas superficies estuviesen afectadas en pacientes jóvenes activos mediante la artroplastia total de Scheker ${ }^{74}$. 


\section{BIBLIOGRAFÍA:}

1. Gomar F. Traumatismos de la unidad radiocubital distal. En: Traumatología. Miembro Superior. Valencia: Edit. Fundación García Muñoz, 1983: 1440-83.

2. Colles A. On the fracture of the carpal extremity of the radius. Edinburgh Med Surg J, 1814; 10: 182-6.

3. Irisarri C. Controversias en el tratamiento de las fracturas de la extremidad distal del radio. Patol Ap Locomotor. Madrid: Fundación Mapfre, 2007; 5 (Supl II): 34-40.

4. Fernández DL, Jupiter JB. The fracture of the distal end of the radius: an historical perspective. En: Fractures of the Distal Radius. New York: Ed. Springer-Verlag, 1996: 1-21.

5. Petit JL. L'Art de Guérir les Maladies de l'Os. París: L. d'Houry, 1705.

6. Pouteaut C. Oeuvres Posthumes de M Pouteaut. Mémoire, contenant quelques réflexions sur quelques fractures de l'avant-bras sur les luxations incomplettes du poignet et sur le diastasis. París: Ph-D Pierres, 1783.

7. Dupuytren G. On the Injuries and Diseases of Bone. Translated by FG Clark. Londres: Sydenham Society, 1847.

8. Malgaigne J. Treatise of Fractures. Translated by J. Packard. Philadelphia: JB Lippincott, 16, 1859.

9. Goyrand G. Mémoire sur les fractures de léxtrémité inférieure du radius qui simulent les luxations du poignet. Gaz Méd, 1832; 3: 664-7.

10. Smith RW. A treatise on fractures in the vicinity of joints and on certain forms of accidental and congenital dislocations. Dublin: Hodges and Smith, 1847.

11. Barton JR. Views and treatment of an important injury of the wrist. Medi Examiner, 1838: 1: 365-8.

12. López Piñero JM. La Medicina en la Historia. Madrid: La Esfera de los Libros, 2002.
13. Lenoble E, Dumontier C. Fracture de l'extremité distal des deux os de l'avant-bras chez l'adulte. Encycl. Med. Chir. Appareil locomoteur, 14-045-B-10, 1997, 14.

14. Meine J. Complicaciones precoces y tardías de la fractura de Colles. En: Buck-Gramcko D, Nigst H. (Eds). Fracturas del Extremo Distal del Radio: Tratamiento y Complicaciones. Barcelona: Edit. Áncora, 1991: 59-67.

15. O'Neill TW, Cooper C, Finn JD, et al. Incidence of distal forearm fracture in British men and women. Osteoporosis Int, 2001; 12: 555-8.

16. Falch JA. Epidemiology of fractures of the distal forearm in Oslo, Norway. Acta Ortop Scand, 1983; 54: 291-5.

17. Ahlborg HG, Johnell O, Turner $\mathrm{CH}$, et al. Bone loss and bone size after menopause. N Engl J Med. 2003; 349: 327-34.

18. Fernandez DL. Fractures of the distal radius. Operative treatment. Instr Course Lect, 1993; 42: 73-88.

19. Pechlaner S, Kathrein A, Gabl $M$, et al. Distal radius fractures and concomitant injuries: experimental studies concerning pathomechanisms. J Hand Surg B, 2003; 28: 609-16.

20. Castaing J, et le Club des Dix. Les fractures récentes de l'extrémité inférieure du radius chez l'adulte. Rapport de la $39^{a}$ Réunion annuelle de La SOFCOT. Rev Chir Orthop, 1964; 50: 581-666.

21. Fernández DL. Avant-bras segment distal. En: Müller ME, Nazarian S, Koch P (Eds). Classification $\mathrm{AO}$ des Fractures des Os Longs, Berlin: Edit SpringerVerlag 1987: 106-15.

22. Müller ME, Nazarian $S$, Koch $P$, et al. The Comprehensive Classification of Fractures of Long Bones. Berlin: Edit SpringerVerlag, 1990.

23. Toledo LFQ, Albertoni WM, Faloppa F. Tratamento das fraturas do rádio distal pela técnica de De Palma modificada. Rev Iberoamer Cir Mano, 2000; 27: 22-7.

24. Delgado PJ, Fuentes A, Martínez de Albornoz P, et al. Reducción indirecta y fijación percutánea en fracturas del radio distal. Patol Ap Locomotor, Madrid: Fundación Mapfre, 2007; 5 (Supl II): 56-63.

25. Pfeiffer KM. Clasificación e indicaciones terapéuticas de las fracturas distales del antebrazo. En: Buck-Gramcko D, Nigst H. (Eds). Fracturas del Extremo Distal del Radio: Tratamiento y Complicaciones. Barcelona: Edit. Áncora, 1991: 15-25.

26. Rikli DA, Regazzoni P. Fractures of the distal end of the radius treated by internal fixation and early function: a preliminary report of 20 cases. J Bone Joint Surg Br, 1996; 78: 588-92.

27. Stein A, Katz S. Stabilization of conminuted fractures of the distal inch of the radius: percutaneus pinning. Clin Orthop, 1975; 108: 174-81.

28. De Palma AF. Conminuted fractures of the distal end of the radius treated by ulnar pinning. $J$ Bone Joint Surg Am, 1952; 34: 651-62.

29. Mortier JP, Kuhlmann JN, Richet $\mathrm{C}$, et al. Brochage horizontal cubito-radial dans les fractures de l'extrémité du radius comportant un fragment postéro-interne. Rev Chir Orthop, 1986, 72: 567-71.

30. Rayhack JM. The history and evolution of percutaneous pinning of displaced distal radius fractures. Orthop Clin North Am, 1993; 24: 287-300.

31. Kapandji A. Le double embrochage intra-focal des fractures de l'extrémité inférieure du radius. Communication aux Journées Franco-Italiennes de Bellagio, 1973. Citado por Kapandji Al. Ostéosynthèse des fractures récentes de l'extrémité inférieure du radius chez l'adulte. Cahiers d'enseignement de la SOFCOT $n^{\circ}$ 46. Conférences 
d'enseignement 1994. Paris: Expansion Scientifique Française, 1994: 19-39.

32. Kapandji A. Ostéosynthèse par double embrochage intra-focal: traitement fonctionnel des fractures non articulaires de l'extrémité inférieure du radius. Ann Chir. 1976, 30: 903-8.

33. Goyeneche I, Torre I. Tratamiento de las fracturas de la extremidad distal del radio según el método de Kapandji. Revisión de 128 casos. Rev Ortop Traum. 1996, 40: 5-9.

34. Py F. Traitement des fractures récentes de l'extrémité inférieure du radius par ostéosynthèse percutanée suivie de mobilisation inmédiate du poignet. À propôs de 600 cas. Thèse de Medicine, Tours 1982.

35. Sato O, Aoki M, Kawaguchi S, e e. Antegrade intramedullary K-wire fixation for distal radial fractures. J Hand Surg Am, 2002; 27: 707-13.

36. Vidal J, Buscayret G, Fischbach $\mathrm{C}$, et al. Une méthode originale dans le traitement des fractures conminutives de l'extrémité inférieure du radius: Le taxis ligamentaire. Acta Orthop Belg, 1977; 43: 781-9.

37. Böhler L. Traitement des Fractures. Paris: Edit. Flammarion, 1944.

38. Ledoux A, Rauis A, Van der Ghinst M. L'embrochage des fractures inferieures du radius. Rev Chir Orthop, 1973; 59: 427-38.

39. Surer P, Ruelle JP, Tiesse J. Traitement des fractures de l'extrémité inférieure du radius par la méthode de Ledoux. Ann Orthop Ouest, 1981; 13: 65-70.

40. Dujardin C, Redreau B, Paillault C, et al. Traitement des fractures graves de l'extrémité inférieure du radius par embrochage bipolaire (technique de Ledoux). Ann Orthop Ouest, 1981; 13: 71-7.

41. Irisarri C. Alcocer L. Tratamiento por tracción bipolar de las fracturas complejas de la extremidad distal del radio. Avances, 1978; 8: 90-109.
42. Jupiter JB, Ring D, Weitzel PP. Surgical treatment of redisplaced fractures of the distal radius in patients older than 60 years. J Hand Surg Am, 2002; 27: 714-23.

43. Alfie VA, Boretto JG, Donndorff AG, et al. Resultados radiológicos obtenidos con la utilización de una placa palmar bloqueada en las fracturas intraarticulares del radio distal en pacientes mayores de 60 años. Rev Iberamer Cir Mano, 2008; 36: 42-9.

44. Orbay JL, Fernández DL. Volar fixation for dorsally displaced fractures of the distal radius: a preliminary report. J Hand Surg Am, 2002; 27: 205-15.

45. Orbay JL, Badía A, Indriago IR, et al. Manejo de la fractura dorsal del radio distal utilizando la placa DVR. Rev Iberoamer Cir Mano, 2001; 28: 28-35.

46. Chiang PP, Roach S, Baratz $\mathrm{ME}$, Failure of a retinacular flap to prevent dorsal wrist pain after titanium $\mathrm{Pi}$ plate fixation of distal radius fractures. $J$ Hand Surg Am, 2002; 27: 724-8.

47. Imatani J, Noda T, Morito $Y$, et al. Minimally invasive plate osteosynthesis for comminuted fractures of the metaphysis of the radius. $\mathrm{J}$ Hand Surg $\mathrm{Br}$, 2005; 30: 220-5.

48. Whipple TL. Arthroscopy Surgery. $1^{a}$ ed. Philadelfia: Lippincott Company, 1992.

49. Del Cerro M, De las Heras J, García D, et al. Utilidad de la artroscopia en el tratamiento de las fracturas de la extremidad distal del radio. Patol Ap Locomotor. Madrid: Fundación Mapfre, 2007; 5 (Supl II): 64-71.

50. Ombrédanne L. L'ostéosynthèse temporaire chez les infants. Presse Méd, 1929; 52: 845-8.

51. Rikli DA, Honigmann P, Babst $\mathrm{R}$, et al. Intrarticular pressure measurement in the radioulnocarpal joint using a novel sensor: In vitro and in vivo results. J Hand Surg Am, 2007; 32: 6775.

52. Margaliot Z, Haase SC, Kotsis SV, et al. A metaanalysis of outcomes of external fixation versus plate osteosynthesis for unstable distal radius fractures. $J$ Hand Surg Am, 2005; 125: 507-14.

53. Arora J, Malik AC. External fixation in conminuted, displaced intrarticular fractures of the distal radius: is it sufficient? Arch Orthop Trauma Surg, 2005; 125: 536-40.

54. Capo JT, Rossy W, Henry P, et al. External fixation of distal radius fractures $\mathrm{J}$ Hand Surg Am, 2009; 34: 1605-11.

55. McQueen MM. Non-spanning external fixation of the distal radius. Hand Clin, 2005; 21: 37580.

56. Krishnan J, Chipchase LS, Slavotinek J. Intraarticular fractures of the distal radius treated with metaphyseal external fixation. Early results. J Hand Surg Br, 1998; 23: 396-9.

57. Gradl G, Jupiter JB, Gierer $\mathrm{Ph}$, et al. Fractures of the distal radius treated with a nonbridging external fixation technique using multiplanar k-wires. $J$ Hand Surg Am, 2005; 30: 960-8.

58. Huber FX, Hillmeier J, Herzog $L$, et al. Open reduction and palmar plate-osteosynthese in combination with a nanocrystalline hidroxyapatite spacer in the treatment of comminuted fractures of the distal radius. J Hand Surg Br, 2006; 31: 298-03.

59. Böhler J. Tratamiento quirúrgico de las fracturas distales del radio. En: Buck-Gramcko D, Nigst H. (Eds). Fracturas del Extremo Distal del Radio: Tratamiento y Complicaciones. Barcelona: Edit Áncora, 1991: 35-50.

60. McQueen MM, Hajducka C, Court-Brown CM. Redisplaced unstable fractures of the distal radius. J Bone Joint Surg Br, 1996; 78: 404-9.

61. Del Piñal F, García-Bernal FJ, Delgado J, et al. Técnica para el tratamiento de la malunión intraarticular del radio median- 
te osteotomías con control artroscópico. Rev Iberoamer Cir Mano, 2005; 33: 38-43.

62. Lluch Homedes A: El Síndrome del Túnel Carpiano. Barcelona: Edit. Mitre, 1987.

63. Celester G, Castro A, Seoane JL. Síndrome del Túnel Carpiano de origen secundario. Rev Iberoamer Cir Mano, 2008; 36: 20-30.

64. Meine J. Complicaciones precoces y tardías de la fractura de Colles. En: Buck-Gramcko D, Nigst H. (Eds). Fracturas del Extremo Distal del Radio: Tratamiento y Complicaciones. Barcelona: Edit. Áncora, 1991: 59-67.

65. García-Elías M. Lesiones asociadas a las fracturas de la extremidad distal del radio. Patol Ap Locomotor. Madrid: Fundación Mapfre, 2007; 5 (Supl II): 22-5.
66. Zechner W. Fractura del extremo distal del radio. Tipos y lesiones asociadas. En: BuckGramcko D, Nigst H. (Eds). Fracturas del Extremo Distal del Radio: Tratamiento y Complicaciones. Barcelona: Edit. Áncora, 1991: 51-8.

67. Lindau T. Treatment of injuries to the ulnar side of the wrist occurring with distal radial fractures. Hand Clin, 2005; 21: 41725.

68. Ring D, McCarty LP, Campbell $\mathrm{D}$, et al. Condylar blade plate fixation of unstable fractures of the distal ulna associated with fracture of the distal radius. J Hand Surg Am, 2004; 29: 1039.

69. Mulford JS, Axelrod T. Traumatic injuries of the distal radioulnar joint. Orthop Clin North America, 2007; 38: 289-97.
70. Tsai P, Paksima N. The distal radioulnar joint. Bull NY Hosp Jt Dis, 2009; 67: 90-6.

71. Sammer DM, Shah HM, Shauver MJ, et al. The effect of ulnar styloid fractures on pacientrated outcomes after volar locking plating of distal radius fractures. J Hand Surg Am, 2009; 34: 1595-602.

72. Souer JS, Ring D, Matschke S, et al. Prospective ORIF Distal Radius Study Group. J Bone Joint Surg Am, 2009; 91: 830-8.

73. Logan AJ, Lindau TR. The management of the distal ulnar fractures in adults: a review of the literature and recommendations for treatment. Strategies Trauma Limb Reconstr, 2008; 3: 49-56.

74. LR Scheker, Breyer JM. Artroplastia total de la articulación radiocubital distal. Rev Iberoamer Cir Mano, 2007; 35: 7-13. 